\title{
ArcheoSciences
}

Revue d'archéométrie

$40 \mid 2016$

Varia

\section{Étude de provenance et implications économico- culturelles des parures vitreuses et résineuses du Bronze moyen de l'abri 1 de Campu Stefanu (Sollacaro, Corse-du-Sud)}

Provenance studies and economico-cultural implications of Middle Bronze Age vitreous and resinous ornaments from from Campu Stefanu, shelter 1 (Corse-duSud)

Kewin Peche-Quilichini, Joseph Cesari, Franck Leandri, Ludovic BellotGurlet, Eleonora Canobbio, Bernard Gratuze, Céline Leandri et Céline Paris

\section{CpenEdition}

Journals

Édition électronique

URL : https://journals.openedition.org/archeosciences/4759

DOI : $10.4000 /$ archeosciences. 4759

ISBN : 978-2-7535-5384-2

ISSN : 2104-3728

Éditeur

Presses universitaires de Rennes

Édition imprimée

Date de publication : 30 décembre 2016

Pagination : 65-81

ISBN : 978-2-7535-5382-8

ISSN : 1960-1360

Référence électronique

Kewin Peche-Quilichini, Joseph Cesari, Franck Leandri, Ludovic Bellot-Gurlet, Eleonora Canobbio, Bernard Gratuze, Céline Leandri et Céline Paris, «Étude de provenance et implications économicoculturelles des parures vitreuses et résineuses du Bronze moyen de l'abri 1 de Campu Stefanu (Sollacaro, Corse-du-Sud) », ArcheoSciences [En ligne], 40 | 2016, mis en ligne le 30 décembre 2018, consulté le 25 février 2022. URL : http://journals.openedition.org/archeosciences/4759; DOI : https:// doi.org/10.4000/archeosciences.4759 


\title{
Étude de provenance et implications
}

\section{économico-culturelles des parures vitreuses}

\author{
et résineuses du Bronze moyen de l'abri 1 \\ de Campu Stefanu (Sollacaro, Corse-du-Sud)
}

\author{
Provenance Studies and Economico-Cultural Implications of Middle Bronze Age \\ Vitreous and Resinous Ornaments from Campu Stefanu, Shelter 1 (Corse-du-Sud)
}

\author{
Kewin Peche-Quilichini ${ }^{a}$, Joseph Cesari ${ }^{b}$, Franck Leandri ${ }^{c}$, \\ Ludovic Bellot-Gurlet ${ }^{d}$, Eleonora Canobbio ${ }^{d}$, Bernard Gratuze ${ }^{e}$, \\ Céline LEANDrI ${ }^{\mathrm{c}}$ et Céline PARIS ${ }^{\mathrm{d}}$
}

\begin{abstract}
Résumé : Les fouilles du site de l'âge du Bronze de Campu Stefanu ont été réalisées entre 2005 et 2011. Elles ont concerné une maison construite durant l'âge du Bronze ancien et un abri naturel. Cet abri à révélé une stratigraphie s'étendant du Mésolithique à l'âge du Fer. Dans ses niveaux de la fin du Bronze moyen, les éléments d'un collier constitué de perles vitreuses et résineuses ont été mis au jour. Ces niveaux sont datés par radiocarbone du XIII ${ }^{e}$ siècle $\mathrm{BC}$. Les perles résineuses montrent une certaine variabilité typologique dont les formes peuvent être rattachées à des éléments égéens de l'Helladique récent. Les analyses par spectroscopies Infrarouge et Raman ont permis d'identifier de l'ambre originaire de la Baltique. L'analyse des perles en verre par LA-ICP-MS a mis en évidence une composition correspondant à une origine mésopotamienne du verre brut employé pour leur fabrication. La reconnaissance d'origines exotiques pour cet assemblage exceptionnel permet d'alimenter le débat sur les contacts entre les bassins occidentaux et orientaux de la Méditerranée durant la seconde moitié du II ${ }^{e}$ millénaire av. J.-C.
\end{abstract}

\begin{abstract}
Archaeological excavations at the Bronze Age settlement of Campu Stefanu (Corsica) have been conducted between 2005 and 2011. These excavations investigated a house built during the Early Bronze Age and a natural shelter with a stratigraphy dating from the Mesolithic to the Iron Age. In the shelter, the Middle Bronze Age levels yielded a necklace made of vitreous and resinous beads. A radiocarbon dating showed these artifacts were deposited during the $13^{\text {th }}$ century BC (last part of the Middle Bronze Age). The range of shapes identified for the resinous artifacts shows similarities with forms diffused in the Aegean area during the Late Helladic period. Their analysis by Infrared and Raman spectroscopies have identified amber of Baltic origin. Glass beads, analysed using LA-ICP-MS, have exhibited an elemental composition indicating a Mesopotamian origin of the raw glass. The identification of an Eastern provenance for this exceptional assemblage is fueling the debate concerning the place of Corsica in the exchange networks linking up the Eastern and Western Mediterranean basins during the second part of the $2^{\text {nd }}$ millennium $B C$.
\end{abstract}

Mots clés : perles, Corse, Bronze moyen, étude de provenance, ambre, verre.

Keywords: beads, Corsica, Middle Bronze Age, provenance study, amber, glass.

a INRAP Méditerranée; ASM, Archéologie des Sociétés Méditerranéennes, UMR 5140, Université de Montpellier 3, CNRS, MCC, 34000 MONTPELLIER, France. (baiucheddu@gmx.fr)

${ }^{b}$ LAMPEA, UMR 7269, Aix Marseille Université, CNRS, MCC, 13094 AIX-EN-PROVENCE, France. (cesari.joseph@gmail.com)

' DRAC-SRA de Corse; TRACES, UMR 5608, Université Toulouse - Jean Jaurès, CNRS, MCC - 5 allée Antonio-Machado, 31058 ToUlOUSE, France. (franck.leandri@culture.gouv.fr) (celine.leandri@culture.gouv.fr)

¿Sorbonne Universités, MONARIS "De la MOlécule aux NAno-objets : Réactivité, Interactions et Spectroscopies ", UMR 8233, UPMC-CNRS, Université Pierre et Marie Curie Paris 6, 75252 PARIS, France. (ludovic.bellot-gurlet@upmc.fr) (eleonora.canobbio@gmail.com)(celine.paris@upmc.fr) 'IRAMAT-CEB, UMR 5060, Université d'Orléans, CNRS, 45071 ORLÉANS, France. (gratuze@cnrs-orleans.fr) 


\section{INTRODUCTION}

L'investigation archéologique du site de Campu Stefanu (Sollacaro, Corse-du-Sud) a été menée entre 2005 et 2011 par J. Cesari, F. Leandri et P. Nebbia. Les fouilles ont permis de reconnaître un habitat ouvert occupé entre la fin du Bronze ancien et la fin du Bronze moyen, soit entre 1800/1700 et 1200 av. J.-C., matérialisé par des maisons implantées sur des terrassements artificiels et par un petit nombre de structures circulaires en pierre sèche à poteau central, de fonction indéterminée. L'extension des recherches a permis l'étude d'un abri-sous-roche placé en périphérie du village. Son remplissage montre une stratigraphie archéologique presque continue entre le Mésolithique et l'extrême fin de l'âge du Fer, notamment caractérisée par la superposition de niveaux sépulcraux. C'est dans le niveau de la fin du Bronze moyen, qui accueillait vraisemblablement un contexte funéraire, qu'un lot de perles en connexion a été exhumé. Au vu des données disponibles dans l'île, les matériaux vitreux et résineux constituant ce collier confèrent à l'ensemble une origine exogène et offrent, pour la première fois sur l'île, un référentiel comparatif avec des éléments similaires et contemporains découverts en Sardaigne, également dans des sépultures. La chronologie, assurée par une datation radiométrique et par l'environnement matériel, est fixée autour du XIII ${ }^{e}$ siècle av. J.-C. L'approvisionnement en produits résineux et vitreux ne pouvant se faire, à cette haute époque, que par l'intermédiaire de réseaux d'échanges sur de longues distances, des analyses de ces matériaux ont été réalisées dans le but de préciser leur origine géographique avant de tenter d'en estimer les possibles vecteurs causaux.

La présente contribution détaille les résultats de ces études de provenance et discute les interprétations culturelles et économiques qui en découlent dans un contexte régional et méditerranéen.

\section{Le site de Campu Stefanu}

\section{Aspects géographiques}

Le site pré- et protohistorique de Campu Stefanu (Cesari et al., 2011; 2012a; 2012b) est localisé à proximité de la rive nord du golfe du Valincu, dans le sud-ouest de la Corse (figure 1). Il occupe une colline de $94 \mathrm{~m}$ d'altitude, à $500 \mathrm{~m}$ au sud d'un important gué du fleuve Taravu. L'intérêt de ce gisement vient du choix de sa localisation qui ne s'inscrit pas dans une position défensive, contrairement aux autres complexes préhistoriques voisins, mais est clairement asso-

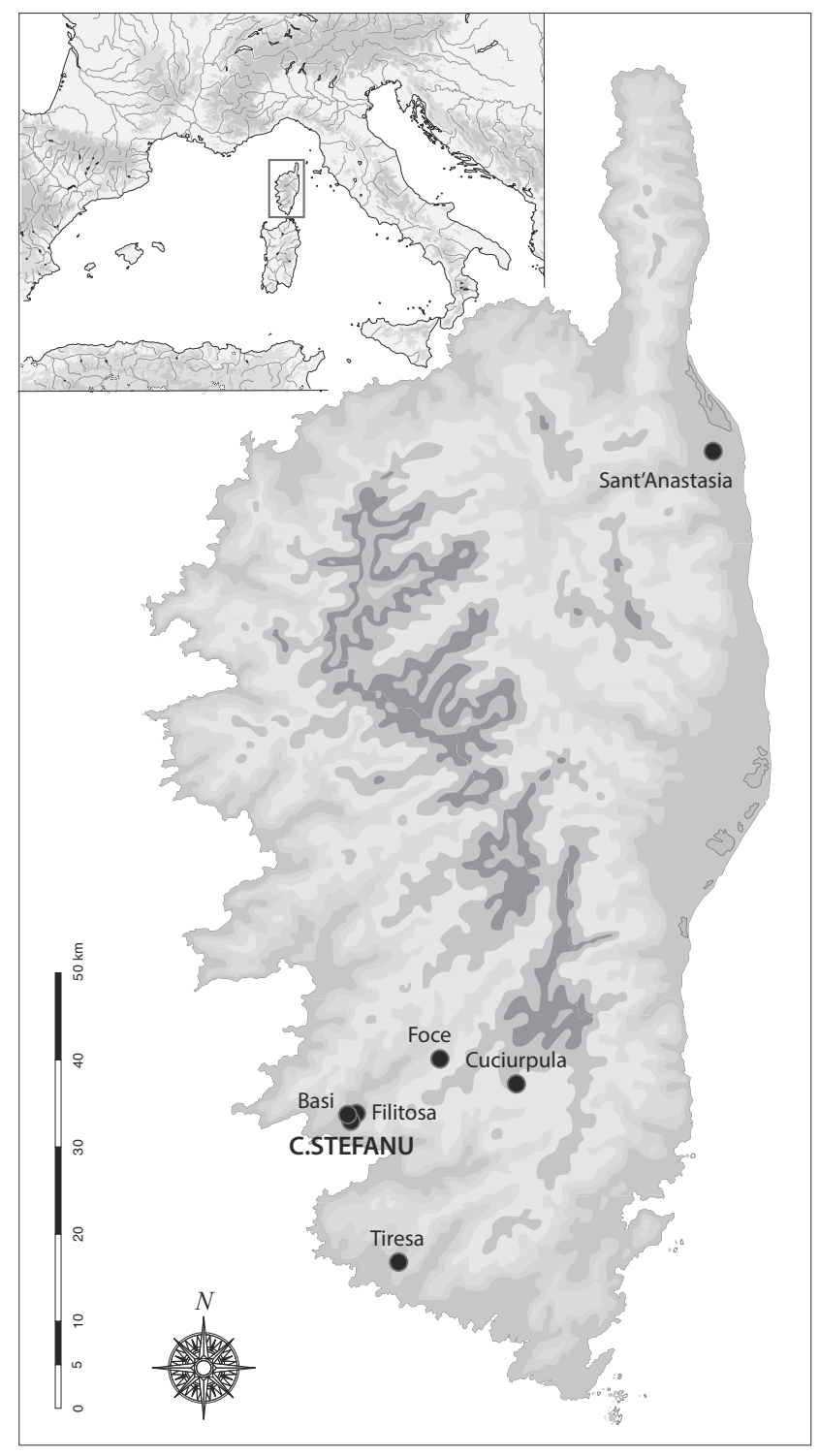

Figure 1 : Carte de la Corse et localisation des sites corses mentionnés dans le texte (DAO : K. Peche-Quilichini).

Figure 1: Map of Corsica and location of Corsican sites mentioned in the text.

cié au franchissement du fleuve. Campu Stefanu est placé sur un axe privilégié qui empruntait les zones collinaires et conduisait vers la haute vallée.

\section{Structuration de l'habitat}

Campu Stefanu compte plusieurs structures bâties à l'aide du granite local. Ces constructions sont de divers types : simples murs de soutènement, arases de bâtiments aux plans variés (elliptique, circulaire, quadrangulaire), 
petits édifices circulaires qui disposaient d'un toit conique. La construction la plus représentative du site, l'habitation 2, est de plan elliptique $(16 \times 4,5 \mathrm{~m})$ et conserve encore un chevet en abside. Le mobilier est homogène et datable entre la seconde moitié du Bronze ancien et la fin du Bronze moyen. Les derniers temps de l'occupation de cette maison sont donc contemporains de l'utilisation sépulcrale et du dépôt de parures de l'abri 1, dont il sera question par la suite. La découverte de quatre fragments de creusets (Peche-Quilichini, 2014; Peche-Quilichini $e t$ al., 2014, figure $1, \mathrm{n}^{\circ} 1,2$ et 13), de scories, de débris de bronze et d'un fragment de moule en terre cuite (PecheQuilichini et al., 2014, figure 4, $n^{\circ} 8$ ) témoigne de la pratique in situ de la métallurgie.

\section{L'abri 1}

L'abri 1 (figure 2) est localisé au bas du site, dans une zone compartimentée par des masses rocheuses qui conserve encore les vestiges de divers aménagements anthropiques. Cette cavité est formée d'une boule granitique creuse d'environ $27 \mathrm{~m}^{2}$ d'emprise au sol et correspond à un taffonu (dans le langage vernaculaire, cavité granitique aux reliefs arrondis en raison de l'érosion) dont la forme est caractéristique de l'érosion des granites du sud de la Corse. La cavité interne, d'environ $13 \mathrm{~m}^{2}$, présente deux chambres (locus 1 et 2) aux plafonds voûtés. L'accès peut se faire par trois ouvertures, au nord, à l'ouest et au sud-est. Les travaux archéologiques ont livré une séquence stratigraphique partiellement remaniée, qui a néanmoins permis d'affiner le phasage chronologique des occupations du gisement. Le niveau le plus profond est constitué d'un dépôt sépulcral collectif mésolithique (Courtaud et al., 2014). Après un niveau d'abandon, la cavité sera réoccupée au Néolithique ancien, moyen et récent, puis au Bronze ancien (Cesari et al., 2011; 2012a; 2012b). Au cours de l'âge du Bronze moyen, dont il sera plus particulièrement question ici, le fonctionnement de la cavité est lié à un nouvel usage sépulcral, matérialisé par l'aménagement d'un caisson en dalles de granite. C'est dans cette séquence que les différents éléments de parure ont été exhumés. A la fin de l'âge du Fer, la cavité sera à nouveau réutilisée à des fins funéraires.

\section{Méthodes analytiQues}

\section{Analyses élémentaires des perles vitreuses}

Vingt-quatre perles ou fragments de perle en verre de Campu Stefanu (sur un total de 27 fragments représen-

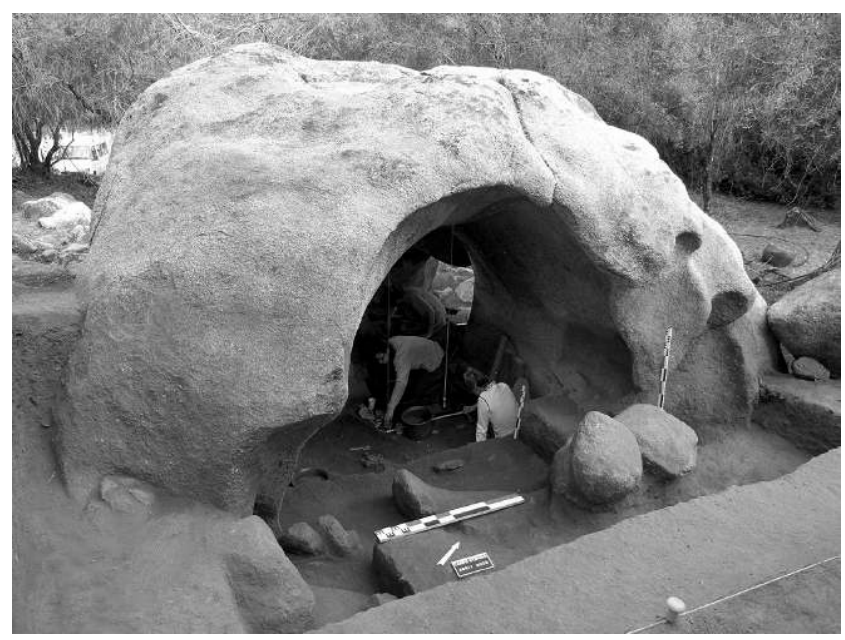

Figure 2 : Vue de l'abri 1 durant la campagne 2008 (photo : J. Cesari).

Figure 2: View of the shelter 1 during the 2008 excavation.

tant au minimum 24 objets ${ }^{1}$ ) ont été analysées par spectrométrie de masse à plasma avec prélèvement par ablation laser (LA-ICP-MS) au Centre Ernest-Babelon à Orléans (IRAMAT, UMR 5060, CNRS/Université d'Orléans). L'instrumentation utilisée comprend un spectromètre de masse (Element XR, Thermo Fisher Instrument) couplé à un système d'ablation laser travaillant à $266 \mathrm{~nm}$ (Nd YAG à fréquence quadruplée, UV microprobe, VG Elemental). L'analyse est réalisée directement sur les objets et ne nécessite pas de préparation particulière des échantillons (Gratuze, 2013, 2014). Ces derniers sont placés dans une cellule d'ablation en quartz avec un ensemble de verres de référence. L'énergie du laser utilisé est de $3 \mathrm{~mJ}$ avec une fréquence de tir de $8 \mathrm{~Hz}$. Le diamètre du cratère d'ablation varie entre 80 et $100 \mu \mathrm{m}$ pour une profondeur d'environ $250 \mu \mathrm{m}$. Un temps de préablation de 20 secondes est programmé avant le début de la mesure du signal, de façon à éliminer la zone de corrosion naturellement présente à la surface des verres. Le signal est ensuite enregistré pendant une période de 50 secondes. La matière prélevée est transportée par un flux d'argon (1 l/ $\mathrm{min})$ vers l'injecteur de la torche à plasma où elle est dissociée, atomisée et ionisée. Les ions sont alors injectés dans le spectromètre de masse où ils sont triés en fonction de leur rapport masse/charge, puis collectés par un multiplicateur d'électrons de type channeltron ou une cage de faraday, en fonction de leur nombre.

Les mesures sont effectuées en mode saut de pic en prenant 4 points par pic pour les mesures effectuées via le chan-

1. La fracturation de certaines perles ne permet pas de trancher sur leur exacte quantité. 
neltron, et 10 points par pic en mode faraday. Le protocole utilisé permet de déterminer les teneurs de 58 éléments au sein de la matrice vitreuse (voir infra). Le balayage en masse du lithium à l'uranium prend environ 2,5 secondes. L'acquisition s'effectue en temps réel, ce qui permet si nécessaire de reconstruire les profils de concentration pour chaque élément mesuré de la surface vers l'intérieur de l'objet. Ceci permet d'une part de vérifier que la totalité de la couche corrodée a été éliminée lors de la pré-ablation, et d'autre part de prendre en compte la présence d'hétérogénéités à l'intérieur du verre (cristaux de matière opacifiante ou grains d'infondus).

Le silicium est mesuré sur l'isotope $29\left({ }^{29} \mathrm{Si}\right)$ et sert d'étalon interne. Les verres de référence NIST 610, Corning B, D et $\mathrm{C}$ ainsi qu'un verre étalon propre au laboratoire (employé pour le dosage du chlore) permettent de calculer les coefficients de réponse de chaque élément (Gratuze, 2014).

Une mesure du bruit de fond est effectuée toutes les dix analyses afin de vérifier l'absence de pollution du flux gazeux. Deux prélèvements par ablation laser sont ensuite effectués pour chaque perle analysée. L'ensemble des verres de référence est analysé une ou plusieurs fois au début et à la fin de chaque séquence analytique, mais aussi régulièrement tout au long de la séquence, afin de détecter et de corriger les dérives éventuelles de l'électronique de mesure.

Le verre de référence Corning A est analysé en tant que matériau inconnu, afin de valider les protocoles expérimentaux (figure 11). Les limites de détection obtenues varient entre 0,1 et $0,01 \%$ pour les éléments majeurs, et entre 20 et $500 \mathrm{ppb}$ pour les autres éléments. La précision des résultats est de l'ordre de 5 à $15 \%$ relatifs selon les éléments et les teneurs mesurés.

\section{Analyses structurales des perles résineuses}

Les analyses par spectroscopie infrarouge ont été réalisées au laboratoire MONARIS (UMR 8233 UPMC-CNRS) en mode micro-ATR (Attenuated Total Reflectance) avec un microscope IRscope II couplé à un spectromètre Equinox 55 (Bruker) équipé d'un détecteur MCT refroidi à l'azote liquide. L'objectif ATR permet l'observation et la sélection de la zone à analyser. Il comporte un cristal de germanium d'une surface de $100 \mu \mathrm{m}$ de diamètre qui est mis en contact avec l'échantillon pour en mesurer l'absorption. Les spectres sont enregistrés sur 200 scans entre 4000 et $600 \mathrm{~cm}^{-1}$ avec une résolution de $4 \mathrm{~cm}^{-1}$.

Les analyses Raman sont effectuées avec une excitation laser dans le proche infrarouge à $1064 \mathrm{~nm}$ (laser Nd-YAG) pour s'affranchir de la forte fluorescence des composés organiques étudiés, qui peut masquer leur diffusion Raman. Le spectro- mètre FT-Raman utilisé (laboratoire MONARIS UMR 8233 UPMC-CNRS) est un Bruker RFS 100/S basé sur un interféromètre de type Michelson associé à un détecteur germanium refroidit à l'azote liquide. Les échantillons sont analysés sans contact par l'intermédiaire d'un microscope et d'un objectif Leica IR x40 (diamètre de la zone analysée $-30 \mu \mathrm{m}$ ). La puissance du laser est ajustée afin de n'induire aucune modification de l'échantillon par chauffage. Les spectres sont enregistrés entre 3500 et $50 \mathrm{~cm}^{-1}$ avec une résolution de $4 \mathrm{~cm}^{-1}$, et un rapport signal sur bruit optimal est obtenu avec l'accumulation de 2000 scans.

\section{4. « Le COLLIER » DE L'ABRI 1 : TyPOLOGIE DES PERLES ET RECONSTITUTION HYPOTHÉTIQUE DE LEUR AGENCEMENT}

La fouille de l'abri 1 a permis de prélever un ensemble en connexion (figure 3) composé d'un séparateur de collier en métal (bronze à l'étain; détermination L. Bellot-Gurlet), de 29 perles résineuses et d'un Nombre Minimal d'Individus (NMI) de 24 (mais vraisemblablement 25 ou 26) perles de verre de teinte bleu turquoise. Ces éléments ont été soumis à des analyses physico-chimiques non-destructives destinés à déterminer la provenance des matériaux.

Si le corpus des perles en verre associe de façon homogène une morphologie en anneau de section plano-convexe à subcirculaire (figures 4 et 5 ) à des gabarits très semblables ( 5 à $10 \mathrm{~mm}$ de diamètre), ce n'est pas le cas du mobilier résineux, pour lequel on remarque une diversité notable, tant du point de vue de la taille des individus que de leur forme. Les perles

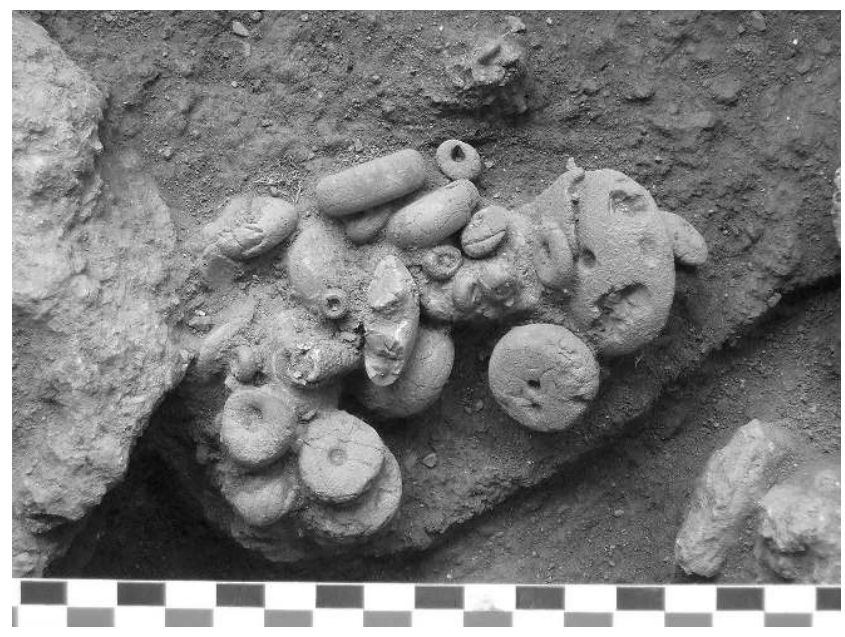

Figure 3 : (Voir planche couleur VIII) Le collier en connexion durant son dégagement (photo : J. Cesari).

Figure 3: (See colour plate VIII) The necklace during the excavation. 
résineuses, de forme sphérique, sub-sphérique ou discoïdale, présentent une perforation centrée ou légèrement décalée, de morphologie et diamètre variables. Cette perforation est de forme cylindrique, probablement forée de façon unidirectionnelle. L'état de dégradation des pièces n'a pas permis de déterminer si la perforation a été réalisée par l'intermédiaire d'un archet. Certaines pièces sont biforées (présence d'un canal plus étroit et excentré à proximité du canal central).
La section des perles varie du cercle au rectangle en passant par l'ovoïde et le plano-convexe. Quelques-unes sont discoïdales; leur tranche est aplatie ou ogivale. Leur diamètre, souvent irrégulier (figure 6), oscille entre 8 et $50 \mathrm{~mm}$. La plus grande est de forme biconique et évoque une fusaïole aplatie. Ses faces portent des creux hémisphériques irréguliers à intervalle régulier, qui pourraient être interprétées comme une décoration altérée. Malheureusement, l'état de
Figure 4 : (Voir planche couleur VIII) Échantillonnage de perles en verre (vue de face), échelle : $5 \mathrm{~mm}$. Les numéros indiquent les références des pièces (photo : B. Gratuze; DAO : K. Peche-Quilichini). Figure 4: (See colour plate VIII) Representative selection of glass beads (front view), scale: $5 \mathrm{~mm}$. Numbers indicate artefact references.

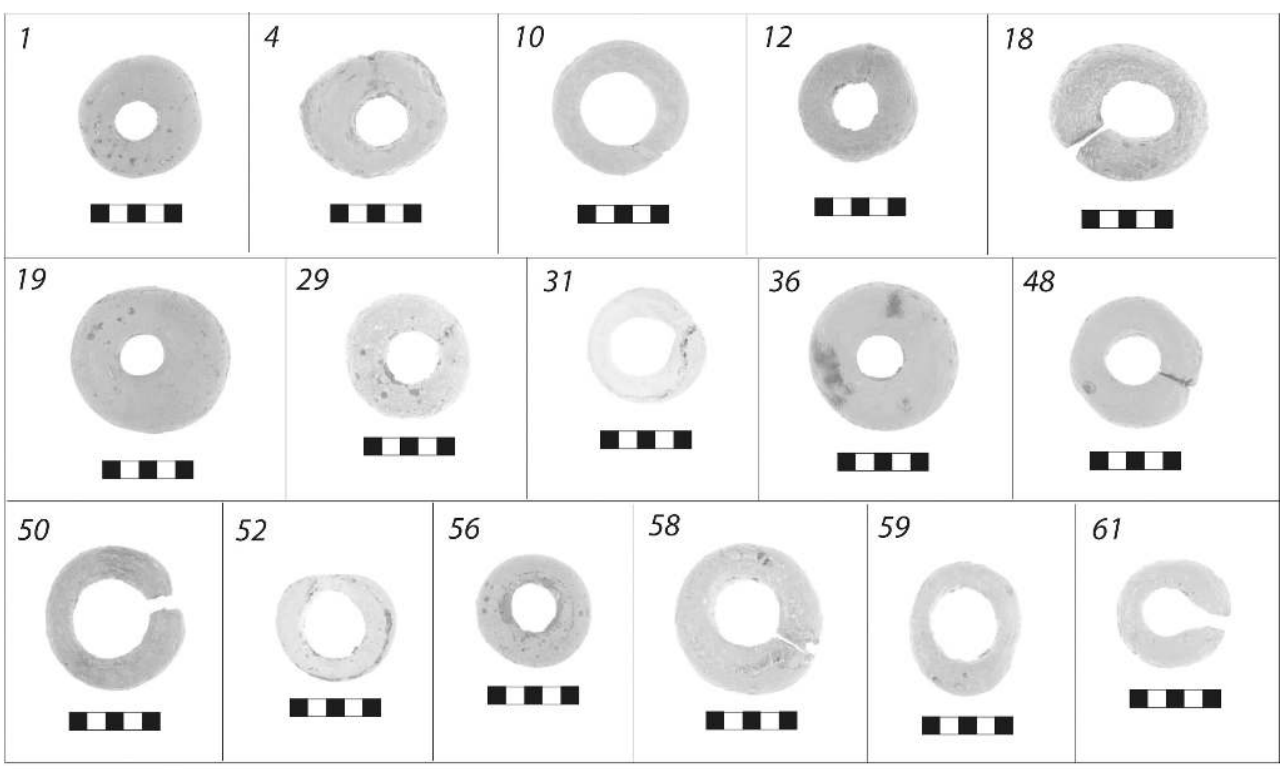

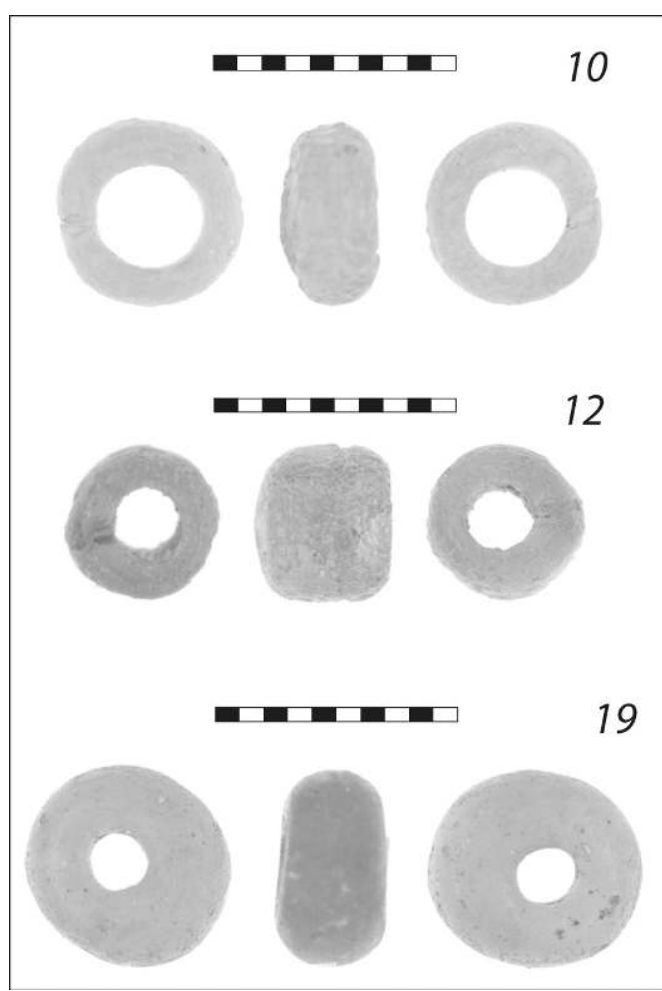

Figure 5: (Voir planche couleur VIII) (Échantillonnage de perles en verre (vue de face et section), échelle : $10 \mathrm{~mm}$. Les numéros indiquent les références des pièces (photo : $\mathrm{B}$. Gratuze; DAO : K. Peche-Quilichini). Figure 5: (See colour plate VIII) Representative selection of glass beads (front view and section), scale: $10 \mathrm{~mm}$. Numbers indicate artefact references.

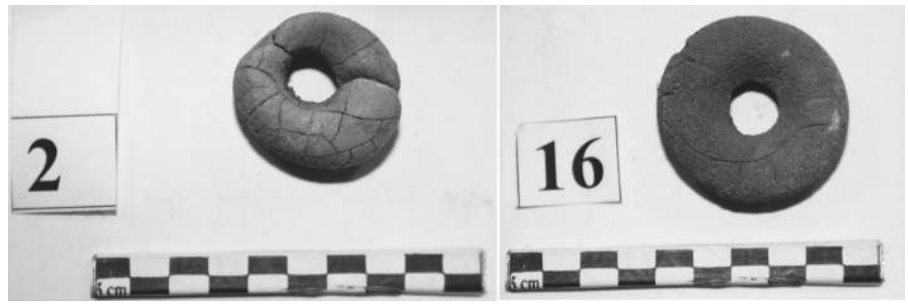

Figure 6: (Voir planche couleur VIII) Perles résineuses ( $\mathrm{n}^{\circ} 2$ et 16) circulaires (photo : C. Leandri).

Figure 6: (See colour plate VIII) Resinous circular beads ( $n^{\circ} 2$ and 16). 
conservation de l'intégralité des pièces a rendu impossible la réalisation de dessins en raison de la rapide décohésion de la surface des objets à la suite de leur exhumation.

Il semble exister une hiérarchie morphométrique entre ces éléments puisque l'une des perles présente donc une forme nettement différente et une taille largement supérieure aux autres, alors que cinq à six individus sont de taille intermédiaire. Cette constatation laisse supposer que l'ensemble formait un collier plutôt qu'un bracelet, même si le cumul des largeurs, estimé à 20-25 cm, donne une longueur assez faible ${ }^{2}$. Le collier pouvait être complété par des éléments en matières périssables, ou uniquement paré dans sa partie pendante. L'alternance des matériaux a été observée à la fouille lors du dégagement de cette parure. Le nombre presque équivalent de perles vitreuses et résineuses laisse supposer que la parure était structurée en deux branches équilibrées, avec les plus grosses perles (résineuses) disposées au centre (figure 7), de part et d'autre d'un séparateur formé par un

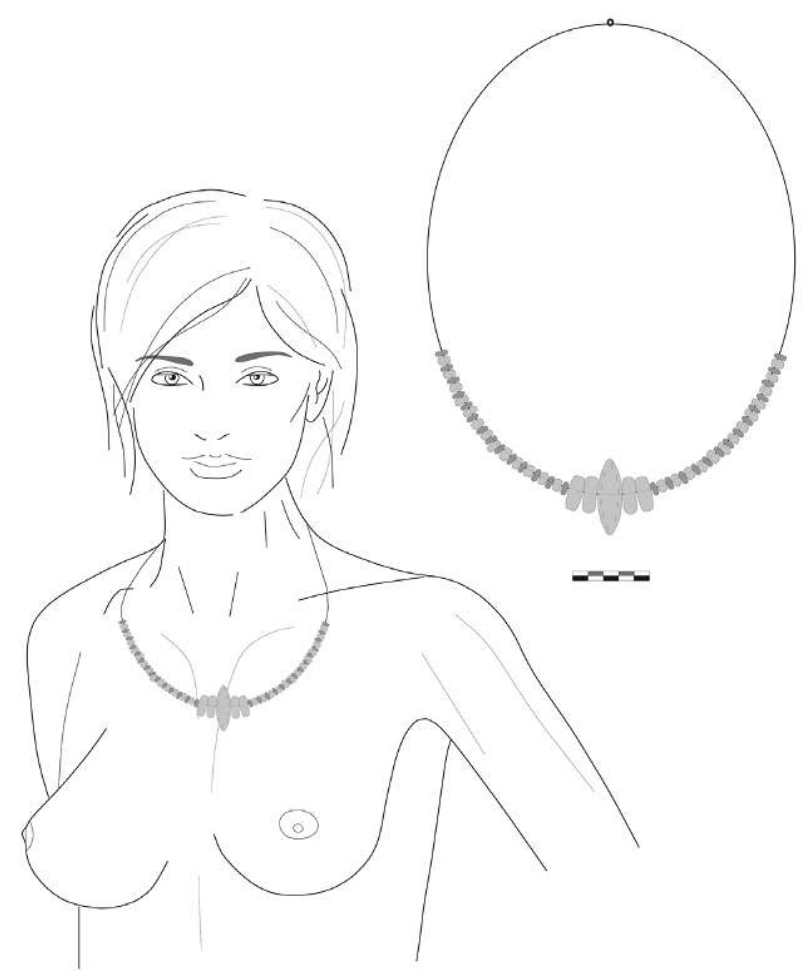

Figure 7 : (Voir planche couleur IX) Hypothèse de reconstitution du collier, échelle : $5 \mathrm{~cm}$ (DAO : K. Peche-Quilichini).

Figure 7: (See colour plate IX) Hypothetical reconstruction of the necklace, scale: $5 \mathrm{~cm}$.

2. Sur cette hypothèse, une confrontation avec des assemblages de perles découverts en contexte mycénien (Mycènes, Thèbes, Peristeria, Vapheio, etc.) pourrait apporter des éléments d'information complémentaires. élément cylindrique torsadé en bronze. L'observation des connexions n'a pas permis d'obtenir d'information complémentaire.

Le lot de perles a été découvert dans un interstice des dalles de pavage de la sépulture qui subsistaient encore à l'aplomb de l'entrée ouest de l'abri. Enfilées sur un lien périssable, les perles sont restées en connexion. Elles s'étaient accumulées et formaient un petit tas piégé dans la légère dépression qui les contenait. Le fait qu'elles n'aient pas été dispersées lors de la destruction de la sépulture permet de supposer qu'il s'agit d'un dépôt intentionnel. La localisation, au niveau de l'entrée de l'abri, renforcerait cette hypothèse. Dans tous les cas, il s'agit d'un enfouissement rapide, autour d'un espace comprimé (bourse?) ou non, caractérisé par la connexion.

\section{ANALYSES PHYSICO-CHIMIQUES DES PERLES : RÉSULTATS ET PREMIÈRES INTERPRÉTATIONS}

\section{Les perles résineuses}

Sur les 29 perles en résine, 26 ont actuellement été analysées afin de déterminer la nature exacte de ces matériaux et de chercher à identifier leur origine botanique et géographique. Trois perles sont encore amalgamées avec un amas de sédiment et elles n'ont donc pas pu être analysées sans prendre le risque d'une fragmentation des objets. À l'occasion d'un éventuel protocole de conservation, la séparation des objets pourra permettre leur analyse. Ces perles sont assez fortement altérées, ce qui a entrainé une décohésion et une fragmentation importante des échantillons, permettant l'observation du cœur de la matière, mais empêchant la réalisation de dessins. Des observations macro- et microscopiques ont permis de mettre en évidence des variations d'aspect et de couleur entre l'extérieur et le cour de l'objet, témoignant de la dégradation de la matière en surface (figure 8) et la présence de parties internes « résineuses " d'aspect brillant.

Lapproche analytique retenue pour caractériser ces échantillons fait intervenir les spectroscopies Raman et infrarouge, qui permettent des mesures non destructives et discriminantes entre les différentes résines (Daher et al., 2010; 2013).

Étant donné le contexte géographique et culturel de ces perles résineuses, une identification comme « ambre » était soupçonnée. Cette résine fossile, largement distribuée en Europe, peut provenir des dépôts principaux des rives de la mer Baltique, mais aussi d'autres formations signalées en France ou en Italie par exemple (du Gardin, 1986; Angelini et Bellintani, 2005; Perrichot et al., 2007; Onoratini et al., 2009; Néraudeau et al., 2012) 

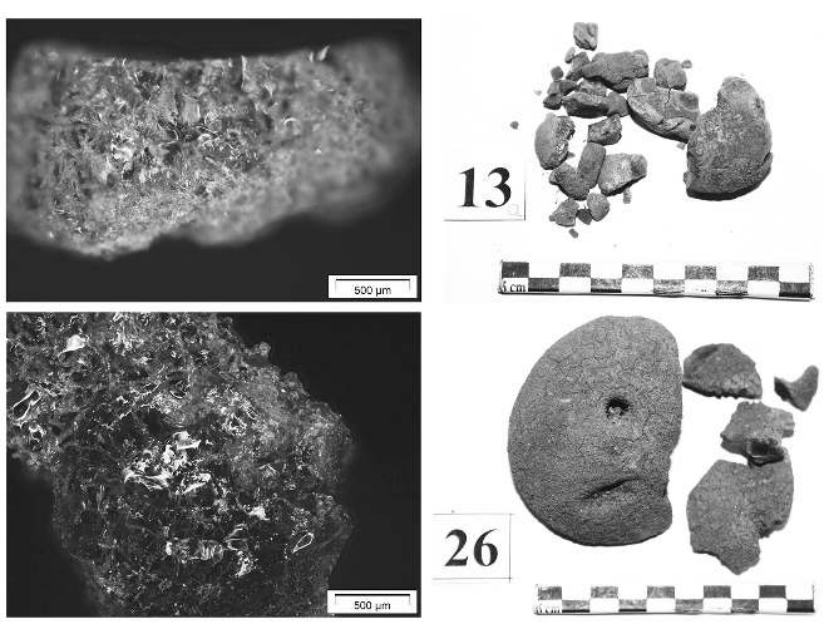

Figure 8 : (Voir planche couleur IX) Vues macro et microscopiques des perles 13 et 26 soulignant leurs hétérogénéités de couleur et leur mauvais état de conservation (photo : L. Bellot-Gurlet, E. Canobbio, C. Paris).

Figure 8: (See colour plate IX) Macro and microscopic views of the beads 26 and 13, which underline their colour heterogeneities and their poor conservation state.

La figure 9 présente des spectres ATR-IR représentatifs de ceux obtenus sur les échantillons archéologiques et celui d'un échantillon de référence d'ambre de la Baltique (succinite). Toutes les perles analysées montrent des spectres ATR-IR caractéristiques de résines fossiles et plus particulièrement un profil spécifique de l'ambre de la Baltique avec un épaulement large entre 1100 et $1330 \mathrm{~cm}^{-1}$, communément appelé Baltic shoulder dans la littérature (Beck et al., 1965; Beck, 1986). Les bandes IR entre 1650 et $1780 \mathrm{~cm}^{-1}$ relatives aux groupes carbonyles évoluent en fonction de la dégradation des résines. Cependant, les spectres obtenus sur les surfaces internes ou externes montrent des signatures spectroscopiques similaires soulignant des structures moléculaires proches.

La figure 10 illustre les spectres Raman obtenus. Bien que moins spécifique que les signatures IR, une forte similitude est observée entre l'échantillon de référence d'ambre de la Baltique et les échantillons archéologiques. Les bandes à 1640 et $1610 \mathrm{~cm}^{-1}$, attribuées aux doubles liaisons carbone-carbone $(\mathrm{C}=\mathrm{C})$, évoluent avec la dégradation des résines, montrant des profils différents entre l'échantillon de référence et les artefacts, mais aussi parfois entre les surfaces internes et externes des perles. Ces signatures Raman semblent donc plus sensibles aux effets du vieillissement et à l'architecture du réseau polymérisé de ces résines fossiles. Une étude ultérieure plus détaillée de ces profils pourrait permettre de documenter plus précisément les états de

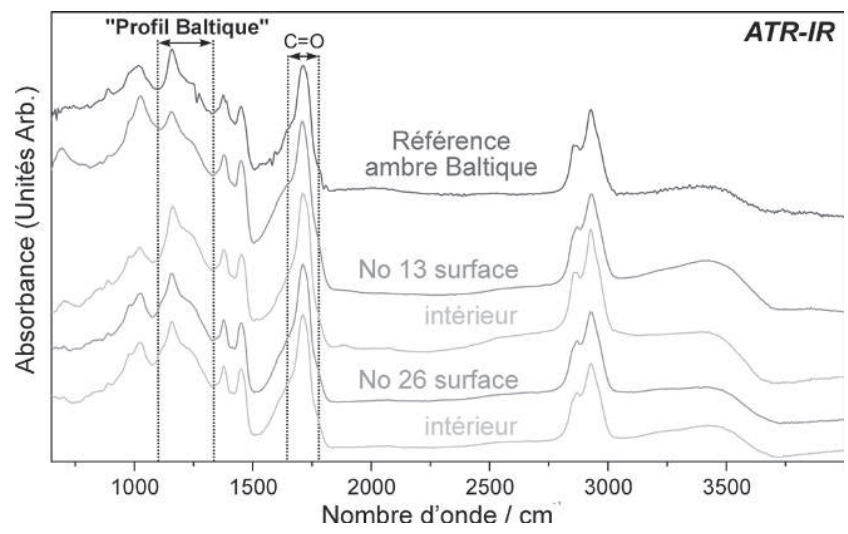

Figure 9 : Spectres ATR-IR des perles 13 et 26, comparés à la signature d'un échantillon de référence d'ambre de la Baltique (DAO : L. Bellot-Gurlet).

Figure 9: ATR-IR representative spectra of beads 13 and 26, for their surfaces or bulk parts, compared to the signature of Baltic reference amber.

conservation de ces échantillons archéologiques (Daher et Bellot-Gurlet, 2013; Pastorelli et al., 2013).

Les résultats obtenus permettent de clairement identifier la nature et la provenance des 26 perles actuellement analysées. Les signatures infrarouges spécifiques documentées dans la littérature pour l'ambre de la Baltique permettent, sans ambigüité, de souligner une provenance du Nord de l'Europe pour les perles retrouvées sur le site de Campu Stefanu.

Du point de vue typologique, pourtant, la forme évoque les perles de Myrsinochori (Beck et Beck, 1995, 122123; Maran, 2013), issues d'ateliers péloponnésiens ${ }^{3}$ des $\mathrm{Xv}^{\mathrm{e}} / \mathrm{XIV}^{\mathrm{e}}$ siècles av. J.-C. (Helladique récent IIB/IIIA1). On sait par ailleurs que les artisans mycéniens de cette époque faisaient venir l'ambre des régions littorales de la Pologne actuelle (Hugues-Brock, 1998, 2005), et que les produits manufacturés étaient disponibles à l'exportation, notamment vers l'ouest (Cultraro, 2007). Selon cette hypothèse, les matériaux découverts à Campu Stefanu auraient donc transité sur plus de $2000 \mathrm{~km}$, du nord au sud, par les fameuses routes de l'ambre, pour être sculptés en contexte mycénien, avant de se retrouver à près de $1200 \mathrm{~km}$ à l'ouest du lieu de production, en Corse, après un nombre inconnu d'étapes intermédiaires, probablement maritimes.

3. Sur le corpus des ambres mycéniens du Péloponnèse, voir : Gaslain et Casanova, 2009, 92-94. 


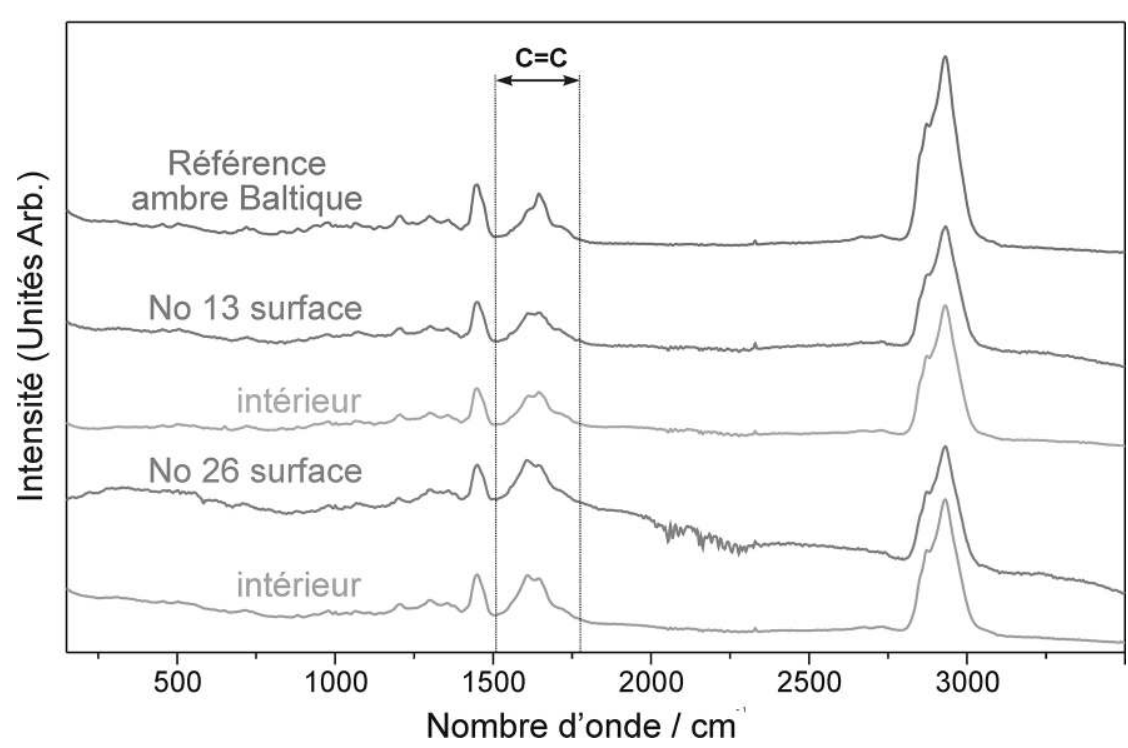

Figure 10 : Spectres FT-Raman des perles 13 et 26 et d'un échantillon de référence d'ambre de la Baltique (DAO : L. BellotGurlet).

Figure 10: FT-Raman representative spectra of beads 13 and 26, for their surfaces or bulk parts, compared to the signature of Baltic reference amber.

\section{Les perles vitreuses}

Si les premiers indices de fabrication du verre sont probablement datés du milieu du III ${ }^{e}$ millénaire, la production de ce matériau à grande échelle a véritablement débuté vers le milieu du II ${ }^{\mathrm{e}}$ millénaire avant notre ère en Mésopotamie et en Égypte (Tite et Shortland, 2008). Il s'agit d'une matière synthétique obtenue par fusion de roches riches en silice (quartz et sables) en présence d'agents fondants (soude ou potasse obtenue à partir de dépôts naturels ou de cendres végétales), destinés à abaisser la température de fusion de la silice. Le calcium et l'aluminium, qui stabilisent la matrice vitreuse et limitent ainsi sa corrosion, entrent dans le mélange soit sous forme d'impuretés, présentes dans les matières premières utilisées, ou de composants ajoutés. Le verre obtenu selon ces protocoles peut être de couleur bleu clair, jaune ou vert, à cause d'impuretés naturelles telles que les oxydes de fer. Des agents colorants (principalement des déchets métallurgiques et des oxydes métalliques de cuivre, de fer ou de manganèse) et/ou opacifiants (composés mixtes à base d'oxydes de plomb, d'antimoine, d'étain et de calcium, voire de cuivre) peuvent être incorporés au mélange pour modifier l'apparence du verre produit. La composition chimique des verres anciens dépend de celles des matières premières disponibles ou sélectionnées et varie en fonction de l'endroit et de l'époque de leur production.

En ce qui concerne la Protohistoire, les objets en verre retrouvés en Europe occidentale et dans le bassin méditerranéen se répartissent au sein de trois grandes familles chimiques selon le type de fondant utilisé (fondants issus de dépôts minéraux ou de cendres de plantes). Différents sous-groupes peuvent y être identifiés en fonction de la source de silice (sables plus ou moins purs ou quartz broyé) et des adjuvants (colorants et opacifiants) employés. En croisant les données issues de l'archéologie et de l'analyse, on observe que ces compositions correspondent à différentes zones de production et se succèdent dans le temps (Billaud et Gratuze, 2002).

Au Bronze moyen, les verres produits en Égypte et en Mésopotamie sont des verres calco-sodiques élaborés par fusion de sables siliceux plus ou moins purs avec des cendres de plantes halophytes (Salicornia sp. ou Salsola sp.). Ce type de verre caractérise la verroterie égyptienne, mésopotamienne et mycénienne jusqu'au $\mathrm{x}^{\mathrm{e}}$ siècle avant notre ère, date à partir de laquelle sa production commence à décliner au profit d'autres recettes (Billaud et Gratuze, 2002). En Europe, cette composition caractérise les perles en verre du Bronze moyen et final importées de Méditerranée orientale (Gratuze et al., 2007; Plouin et al., 2012; Varberg et al., 2015; à paraître). On distinguera cependant les zones de production de verre brut, matière première élaborée au sein d'ateliers dits primaires, dont la localisation est essentiellement reconnue pour cette époque en Egypte et en Mésopotamie, de celles des produits finis, perles et autres objets fabriqués au sein d'officines ou ateliers dits secondaires, dont la répartition, plus vaste, englobe les mondes égyptien, mésopotamien, mais aussi égéen et peut être levantin (Rehren et Pusch, 2005; Bennet, 2008; Tite et al., 2008; Walton et al., 2009; Smirniou et Rehren, 2013; Rehren, 2014). Le matériel en verre retrouvé au sein de l'épave d'Uluburun, composé à la fois de lingots de verre brut et de perles, illustre bien l'organisation, déjà très structurée, de la produc- 
tion et de la commercialisation du verre à l'âge du Bronze (Pulak, 2008). Les études archéométriques récentes (Rehren et Pusch, 2005; Shortland et Eremin, 2006; Shortland et al., 2007; Walton et al., 2009; Jackson et Nicholson, 2010) montrent qu'il est possible de différencier les productions égyptiennes des productions mésopotamiennes sur la base des éléments traces (impuretés) apportées par les matières premières employées (chrome, lanthane, zirconium et titane principalement).

Les résultats obtenus sur les perles en verre de Campu Stefanu (figure 11) montrent que le verre utilisé pour leur fabrication est un verre calco-sodique, avec des teneurs élevées en potasse ( 1,8 à $3,5 \%)$ et magnésie $(4,1$ à $6,0 \%)$ caractéristiques des verres élaborés par fusion d'un sable avec des cendres de plantes halophytes (Salicornia sp. ou Salsola sp.). Ce verre présente des teneurs variables en chaux $(4,3$ à $7,7 \%)$ et alumine $(0,6$ à 1,9\%) qui traduisent l'emploi de sables plus ou moins purs. La forte homogénéité typologique de l'ensemble étudié, qui peut laisser supposer une origine commune de ces perles (même centre de fabrication), contraste donc fortement avec la variabilité de composition observée pour les éléments majeurs, mineurs et traces. Cette dernière souligne en effet que le verre employé ne provient probablement pas d'une seule et même phase de fabrication mais plus certainement de plusieurs bains de fusion, voire éventuellement de différents ateliers primaires. Peu de données sont disponibles à ce jour sur la variabilité de compositions des productions verrières au sein des ateliers de cette époque (Walton et al., 2009; Smirniou et Rehren, 2013). Sur le plan statistique, le lot de parures en verre de Campu Stefanu, qui constitue l'un des plus gros ensembles homogènes analysés à ce jour, est à ce titre un bon indicateur de la variabilité de composition que l'on peut être amené à rencontrer pour les productions des ateliers secondaires de cette époque. Cette variabilité peut être considérée comme le reflet indirect, avec un effet grossissant probable, de celle des productions des ateliers primaires.

La couleur bleue des perles résulte d'une adjonction de cuivre ( $\mathrm{Cu}$ de 0,56 à 1,2\%) sous une forme relativement pure, au bain de fusion. Les éléments traditionnellement associés à ce métal dans les alliages métalliques de l'époque, étain, plomb, voire antimoine et arsenic, sont en effet présents en faibles quantités (teneurs maximales inférieures à $0,01 \%)$ et reflètent l'emploi d'une matière colorante contenant en moyenne plus de 98,5\% de cuivre (figure 11). Les faibles concentrations en étain sont un argument supplémentaire en faveur d'une origine mésopotamienne du verre brut employé pour la fabrication des perles. Les travaux d'Andrew Shortland (Shortland, 2005) sur les verres provenant d'ateliers égyptiens et de sites mésopotamiens montrent en effet que les verriers égyptiens utilisent plutôt, comme matière colorantes, des déchets d'alliages cuivreux, comme le bronze, qui apportent des concentrations notables en étain, tandis que les verriers mésopotamiens utilisent un agent colorant à base de cuivre plus pur.

D'un point de vue technique, on a pu morphologiquement déterminer que toutes les perles ont été mises en forme par pliage du verre ramolli autour d'une tige, pour être ensuite sectionnée par torsion. La morphologie des perles de Campu Stefanu est proche de celle de plusieurs exemplaires provenant de contextes sépulcraux égyptiens datés du milieu du II ${ }^{e}$ millénaire av. J.-C. (Shortland et al., 2007; Nakai et al., 2009), ainsi qu’à de nombreux échantillons découverts sur des sites européens de l'âge du Bronze moyen (Plouin et al., 2012; Gratuze et al., 2013). Néanmoins, l'analyse des pâtes montre (figure 12) que l'origine du verre est vraisemblablement à rechercher en Mésopotamie, même si les perles ont par la suite pu être produites dans la vallée du Nil et diffusées à partir du Delta ou des emporia de la côte levantine.

\section{IMPLiCATIONS CULTURELLES}

Depuis plusieurs années, les travaux sur l'âge du Bronze corse s'étaient orientés principalement vers l'étude des gisements fortifiés, essentiellement sur les torre implantées à l'intérieur des castelli (sites fortifiés, ceinturés par une ou plusieurs enceintes). Les fouilles de Campu Stefanu s'inscrivent dans la perspective de nouvelles recherches sur l'évolution de l'habitat installé en milieu ouvert. Ce site offre en effet l'originalité d'être en rupture avec les installations perchées marquées par leurs aspects défensifs, dont la typologie a été illustrée dans le Taravu par les travaux anciens de R. Grosjean (1958) puis par ceux de J. Cesari (1989a; 1989b; 1992). Ainsi, la fouille de la structure 2, une grande maison de plan elliptique implantée au centre de cet habitat, a mis en évidence des structures jusqu'ici inédites en Corse. Du point de vue matériel, l'étude de ce contexte domestique montre notamment la pratique in situ et probablement quotidienne de la métallurgie du bronze. On doit souligner ici que la parure découverte dans l'abri 1 est contemporaine ${ }^{4} \mathrm{de}$ la dernière phase d'occupation de la structure 2 , interprétée comme une habitation (Cesari et al., 2011).

Cette parure révèle, pour la première fois en contexte fiable, des éléments issus d'une importation à une époque qui

4. Un charbon de bois provenant du niveau d'abandon de la structure 2 et de la terrasse a fourni le résultat suivant : UGAMS 3827, $2970 \pm 25 \mathrm{BP}$ soit 1252-1149 Cal. BC, soit une date calendaire de $1201 \pm 51 \mathrm{BP}$, avec une probabilité de $68 \%$. 


\begin{tabular}{|c|c|c|c|c|c|c|c|c|c|c|c|c|c|c|c|c|c|c|c|}
\hline & $\mathrm{Na}_{2} \mathrm{O}$ & $\mathrm{MgO}$ & $\mathrm{Al}_{2} \mathrm{O}_{3}$ & $\mathrm{SiO}_{2} \mathrm{P}$ & $\mathrm{P}_{2} \mathrm{O}_{5}$ & $\mathrm{~K}_{2} \mathrm{O}$ & $\mathrm{CaO}$ & $\mathrm{Fe}_{2} \mathrm{O}_{3}$ & CuO & $\mathrm{Li}_{2} \mathrm{O}$ & $\mathrm{B}_{2} \mathrm{O}_{3} \quad \mathrm{TiC}$ & $\mathrm{TiO}_{2} \quad \mathrm{~V}_{2} \mathrm{O}_{5}$ & $\mathrm{Cr}_{2} \mathrm{O}_{3}$ & $\mathrm{MnO}$ & $\mathrm{CoO}$ & $\mathrm{NiO}$ & $\mathrm{ZnO}$ & $\mathrm{GaO}$ & $\mathrm{As}_{2} \mathrm{O}_{3}$ \\
\hline CSTF 1 & 15,1 & 4,37 & 1,43 & 68,9 & $0,17 \quad 0,78$ & 3,01 & 4,35 & 0,43 & 1,27 & 51 & 304 & 422 & 19 & 448 & 7,4 & 21 & 32 & 2,5 & 44 \\
\hline CSTF 4 & 17,2 & 4,51 & 0,83 & $68,2 \quad 0$ & $0,18 \quad 0,86$ & 2,44 & 4,28 & 0,55 & 0,76 & 45 & $550 \quad 46$ & 15 & 19 & 458 & 6,4 & 19 & 39 & 1,6 & 14 \\
\hline CSTF 10 & 17,4 & 6,04 & 1,40 & 63,30 & $0,25 \quad 0,72$ & 2,96 & 5,89 & 0,70 & 1,15 & 66 & $379 \quad 55$ & 17 & 25 & 599 & 9,0 & 16 & 44 & 2,9 & 23 \\
\hline CSTF 12 & 17,0 & 5,18 & 1,35 & $65,2 \quad 0$ & $\begin{array}{ll}0,27 & 0,79\end{array}$ & 2,81 & 5,13 & 0,84 & 1,13 & 71 & 466 & 26 & 41 & 529 & 7,2 & 23 & 67 & 2,7 & 23 \\
\hline CSTF 18 & 17,7 & 5,27 & 0,78 & 65,50 & $0,26 \quad 0,69$ & 3,38 & 4,51 & 0,60 & 0,98 & 54 & $567 \quad 58$ & 587 & 31 & 416 & 12 & 16 & 43 & 1,6 & 41 \\
\hline CSTF 19 & 14,6 & 4,38 & 1,40 & $68,2 \quad 0$ & $0,19 \quad 0,57$ & 3,30 & 5,34 & 0,68 & 1,14 & 53 & 528 & 21 & 46 & 528 & 6,3 & 21 & 37 & 2,5 & 29 \\
\hline CSTF 20 & 16,6 & 5,14 & 0,86 & $66,3 \quad 0$ & $0,20 \quad 0,72$ & 3,17 & 5,12 & 0,44 & 1,23 & 47 & 457 & 14 & 28 & 483 & 5,9 & 19 & 37 & 1,3 & 24 \\
\hline CSTF 28 & 19,2 & 5,49 & 1,75 & $61,6 \quad 0$ & $0,31 \quad 0,73$ & 3,28 & 5,36 & 0,91 & 1,09 & 55 & 428 & 25 & 29 & 622 & 5,3 & 20 & 53 & 4,1 & 22 \\
\hline CSTF 29B & 17,9 & 4,71 & $0,56 \quad 6$ & $65,7 \quad 0$ & $0,19 \quad 0,90$ & 3,32 & 5,33 & 0,55 & 0,71 & 76 & 360 & 277 & 16 & 254 & 28 & 12 & 29 & 1,2 & 34 \\
\hline CSTF 31 & 16,6 & 4,58 & 0,84 & $66,3 \quad 0$ & $0,16 \quad 0,82$ & 3,30 & 5,71 & 0,50 & 0,94 & 53 & 333 & 406 & 25 & 541 & 10 & 18 & 39 & 1,4 & 23 \\
\hline CSTF 32 & 16,9 & 4,55 & 0,90 & $65,8 \quad 0$ & $0,17 \quad 0,84$ & 3,39 & 5,79 & 0,50 & 0,93 & 55 & $334 \quad 40$ & 17 & 24 & 539 & 10 & 18 & 40 & 1,4 & 24 \\
\hline CSTF 36 & 15,6 & 4,37 & 1,21 & $65,8 \quad 0$ & $0,15 \quad 0,81$ & 3,18 & 7,08 & 0,39 & 1,19 & 44 & 317 & 15 & 25 & 453 & 5,0 & 17 & 27 & 1,9 & 36 \\
\hline CSTF 45 & 15,0 & 4,26 & 1,64 & $68,2 \quad 0$ & $0,17 \quad 0,62$ & 3,27 & 4,93 & 0,65 & 1,05 & 52 & 491 & 19 & 37 & 503 & 5,9 & 21 & 39 & 2,7 & 31 \\
\hline CSTF 46 & 18,5 & 5,20 & 1,48 & $63,2 \quad 0$ & $0,18 \quad 0,86$ & 3,11 & 5,52 & 0,59 & 1,14 & 56 & $485 \quad 52$ & 524 & 31 & 562 & 10 & 20 & 39 & 2,4 & 25 \\
\hline CSTF 47 & 17,6 & 4,40 & 1,11 & $67,2 \quad 0$ & $0,17 \quad 0,67$ & 2,27 & 4,73 & 0,53 & 1,12 & 41 & 491 & 15 & 46 & 440 & 7,4 & 22 & 35 & 1,5 & 28 \\
\hline CSTF 48 & 16,3 & 5,31 & 0,97 & $66,8 \quad 0$ & $0,15 \quad 0,70$ & 2,68 & 5,24 & 0,44 & 1,19 & 59 & $526 \quad 42$ & 14 & 37 & 457 & 6,4 & 21 & 30 & 1,3 & 15 \\
\hline CSTF 50 & 19,3 & 4,96 & 1,49 & $62,6 \quad 0$ & $0,23 \quad 0,79$ & 3,08 & 5,10 & 0,74 & 1,47 & 72 & $441 \quad 83$ & 21 & 38 & 513 & 8,2 & 19 & 45 & 3,1 & 17 \\
\hline CSTF 51 & 16,9 & 4,58 & 1,06 & $63,9 \quad 0$ & $0,15 \quad 0,82$ & 3,00 & 7,72 & 0,44 & 1,12 & 50 & $332 \quad 57$ & 17 & 33 & 474 & 6,3 & 19 & 30 & 8 & 35 \\
\hline CSTF 52 & 16,1 & 4,58 & 0,88 & $66,8 \quad 0$ & $0,17 \quad 0,83$ & 3,25 & 5,74 & 0,54 & 0,93 & 56 & $341 \quad 48$ & 481 & 25 & 558 & 10 & 19 & 47 & 1,6 & 24 \\
\hline CSTF 56 & 15,5 & 4,11 & 1,90 & $67,8 \quad 0$ & $0,18 \quad 0,65$ & 3,28 & 4,70 & 0,63 & 1,06 & 56 & $482 \quad 66$ & 21 & 33 & 487 & 6,0 & 21 & 34 & 2 & 33 \\
\hline CSTF 57 & 17,9 & 4,68 & 1,04 & $66,4 \quad 0$ & $0,10 \quad 1,02$ & 1,82 & 5,32 & 0,73 & 0,77 & 55 & 252 & 14 & 34 & 256 & 19 & 13 & 34 & 8 & 39 \\
\hline CSTF 58 & 17,5 & 4,89 & 0,82 & $64,3 \quad 0$ & $0,17 \quad 0,89$ & 3,46 & 6,40 & 0,45 & 0,95 & 61 & 360 & 15 & 27 & 540 & 10 & 18 & 35 & 1,4 & 27 \\
\hline CSTF 59 & 18,4 & 4,78 & 1,07 & $65,4 \quad 0$ & $0,16 \quad 0,85$ & 2,43 & 5,08 & 0,51 & 1,10 & 45 & $418 \quad 44$ & 18 & 27 & 471 & 6,6 & 18 & 39 & 2,0 & 45 \\
\hline CSTF 61 & 17,2 & 4,92 & 0,87 & $64,5 \quad 0$ & $0,18 \quad 0,86$ & 3,46 & 6,45 & 0,47 & 0,92 & 53 & 347 & 15 & 25 & 540 & 10 & 18 & 34 & 1,3 & 26 \\
\hline CORN A & 14,3 & 2,48 & 0,92 & 67,1 & $0,1 \quad 0,15$ & 2,91 & 5,04 & 1,11 & 1,21 & 1111 & $1894 \quad 722$ & $7228 \quad 62$ & 31 & 10063 & 31820 & 240 & 529 & 1,1 & 33 \\
\hline & $\mathrm{As}_{2} \mathrm{O}_{3}$ & $\mathrm{Rb}_{2} \mathrm{O}$ & $\mathrm{SrO}$ & $\mathrm{Y}_{2} \mathrm{O}_{3}$ & ${ }_{3} \mathrm{ZrO}_{2} \quad \mathrm{~N}$ & $\mathrm{Nb}_{2} \mathrm{O}_{3}$ & $\mathrm{Ag}$ & $\mathrm{SnO}_{2}$ & $\mathrm{Sb}_{2} \mathrm{O}_{3}$ & $\mathrm{Cs}_{2} \mathrm{O}$ & $\mathrm{BaO}$ & ${ }_{2} \mathrm{O}_{3}$ & $\mathrm{CeO}_{2}$ & $\mathrm{PrO}_{2}$ & $\mathrm{H}_{2} \mathrm{O}_{3}$ & $\mathrm{HfO}_{2}$ & $\mathrm{PbO}$ & $\mathrm{ThO}_{2}$ & $\mathrm{UO}_{2}$ \\
\hline CSTF 1 & 44 & 30 & 310 & 3.4 & 13 & 18 & 16 & 20 & 40 & 0,62 & 50 & 28 & 68 & 0,7 & 6 & 0,39 & 20 & 0,98 & 0,55 \\
\hline CSTF 4 & 14 & 22 & 357 & 3,2 & 14 & 1,0 & 1,3 & 23 & 2,2 & 0,88 & 52 & 3,6 & 13,6 & 0,7 & 3,4 & 0,35 & 12 & 0,64 & 0,51 \\
\hline CSTF 10 & 23 & 24 & 653 & 3,9 & 21 & 1,7 & 2,5 & 27 & 4,6 & 0,90 & 59 & 5,2 & 12,5 & 1,1 & 16,9 & 0,52 & 12 & 1,20 & 0,68 \\
\hline CSTF 12 & 23 & 24 & 460 & 4,6 & 17 & 2,1 & 1,8 & 47 & 2,3 & 0,55 & 68 & 4,3 & 23,0 & 1,4 & 3,7 & 0,39 & 14 & 0,89 & 0,54 \\
\hline CSTF 18 & 41 & 11 & 479 & 2,4 & 10 & 1,0 & 1,6 & 36 & 1,9 & 0,36 & 50 & 2,7 & 10,7 & 0,8 & 2,1 & 0,24 & 17 & 0,52 & 0,35 \\
\hline CSTF 19 & 29 & 19 & 409 & 4,4 & 17 & 1,8 & 0,7 & 82 & 4,1 & 0,48 & 57 & 3,6 & 10,1 & 0,8 & 3,4 & 0,42 & 14 & 1,14 & 0,54 \\
\hline CSTF 20 & 24 & 8,1 & 492 & 3,0 & 14 & 1,0 & 1, & 29 & 2,2 & 0,28 & 50 & 3,0 & 5,9 & 0,7 & 3,3 & 0,35 & 14 & 0,62 & 0,28 \\
\hline CSTF 28 & 22 & 22 & 532 & 5,0 & 22 & 2,0 & 0, & 28 & 2,0 & 0,59 & $\begin{array}{ll}9 & 101\end{array}$ & 5,4 & 19,1 & 1,9 & 6,4 & 0,52 & 16 & 1,41 & 0,81 \\
\hline CSTF 29B & 34 & 10 & 405 & 2,1 & 10 & 0,7 & 0 , & 14 & 8,1 & 0,28 & 28 & 1,9 & 3,7 & 0,4 & 1,7 & 0,24 & 14 & 0,49 & 0,22 \\
\hline CSTF 31 & 23 & 13 & 530 & 3,1 & 15 & 1,0 & 1,8 & 22 & 4,8 & 0,33 & 57 & 2,8 & 6 , & 0,6 & 2,5 & 0,32 & 17 & 0,61 & 0,28 \\
\hline CSTF 32 & 24 & 13 & 545 & 3,2 & 16 & 0 , & 1, & 23 & 4,7 & 0,41 & 58 & 31 & 6,7 & 1,4 & 27 & 0,36 & 16 & 0,65 & 0,28 \\
\hline CSTF 36 & 36 & 16 & 719 & 3,8 & 24 & 1,6 & 1,5 & 48 & 3,4 & 0,32 & 56 & 3,9 & 7,8 & 3,6 & 3,3 & 0,62 & 14 & 1,25 & 0,48 \\
\hline CSTF 45 & 31 & 22 & 353 & 4,2 & 22 & 2,0 & 0,9 & 34 & 5,8 & 0,43 & 62 & 4,3 & 9,7 & 2,4 & 3,6 & 0,56 & 18 & 1,24 & 0,54 \\
\hline CSTF 46 & 25 & 19 & 525 & 3,8 & 18 & 1, & 0 , & 1 & 5 , & 0,45 & 53 & 3, & 7,1 & 0,8 & 3,2 & 0,46 & 15 & 1,03 & 0,48 \\
\hline CSTF 47 & 28 & 8,8 & 320 & 3,2 & 19 & 1,3 & 2 & 29 & 2,5 & 0,24 & 46 & 7 , & 6,2 & 0,7 & 2,8 & 0,44 & 13 & 1,67 & 0,39 \\
\hline CSTF 48 & 15 & 13 & 473 & 3,3 & 15 & 1,0 & 1, & $1 s$ & 2, & 0,33 & 45 & 3, & 5,8 & 0,7 & 2,7 & 0,33 & 12 & 0,62 & 0,22 \\
\hline CSTF 50 & 17 & 24 & 391 & 4,8 & 19 & 2,2 & 0,9 & 45 & 8,2 & 0,59 & 71 & 5,9 & 13,4 & 13,7 & 9,0 & 0,54 & 13 & 1,33 & 0,88 \\
\hline CSTF 51 & 35 & 16 & 775 & 3,7 & 21 & 1,2 & 1,5 & 28 & 3,0 & 0,46 & 56 & 4, & 8,5 & 0,9 & 3,4 & 0,50 & 15 & 0,96 & 0,43 \\
\hline CSTF 52 & 24 & 15 & 543 & 3,2 & 16 & 1,0 & 18,7 & 20 & 4,9 & 0,43 & 58 & 3, & 7 & 0 & 2,7 & 0,36 & 17 & 0,61 & 0,36 \\
\hline CSTF 56 & 33 & 24 & 319 & 4,5 & 23 & 2, & 0,8 & 21 & 7,1 & 0 , & 6 & & 8,8 & & 3, & 0,59 & 17 & 1,40 & 0,64 \\
\hline CSTF 5 & 39 & 16 & 499 & 2,9 & 18 & 1, & 1, & 18 & 11 & 0,46 & 41 & 2, & 5, & 0,6 & 2,5 & 0,43 & 13 & 0,83 & 0,54 \\
\hline CSTF 58 & 27 & 15 & 619 & 3,1 & 15 & 0, & $x_{\prime}$ & 3 & 4,0 & 0,34 & 56 & & 5 , & & 2, & 0,36 & 16 & 0,60 & 0,28 \\
\hline CSTF 59 & 45 & 10 & 435 & 3,5 & 16 & 1,5 & 2 & 41 & 3,4 & 0,34 & 63 & 3,6 & 6,9 & 0,8 & 3,1 & 0,38 & 23 & 0,93 & 0,49 \\
\hline CSTF 61 & 26 & 10 & 619 & 3,3 & 16 & 1,1 & 1,3 & 25 & 4,1 & 0,34 & 56 & 19,2 & 5,8 & 0,7 & 2,7 & 0,38 & 16 & 0,70 & 0,29 \\
\hline CORN A & 33 & 96 & 1002 & $2 \quad 0,5$ & 52 & 0,7 & 19,4 & 1706 & 16671 & 0,26 & $6 \quad 4278$ & 0,4 & 0,4 & 0,0 & 0,1 & 1,04 & 806 & 0,33 & 0,22 \\
\hline
\end{tabular}

Figure 11 : Composition chimique des perles en verre de Campu Stefanu exprimée en pourcentage massique pour les principaux oxydes $\left(\mathrm{Na}_{2} \mathrm{O}, \mathrm{MgO}, \mathrm{Al}_{2} \mathrm{O}_{3}\right.$, $\mathrm{SiO}_{2}, \mathrm{P}_{2} \mathrm{O}_{5}, \mathrm{Cl}, \mathrm{K}_{2} \mathrm{O}, \mathrm{CaO}, \mathrm{Fe}_{2} \mathrm{O}_{3}$, $\mathrm{CuO})$ et en parties par million d'oxydes pour les autres éléments. Les teneurs mesurées pour le verre de référence Corning A (CORN A) sont données à titre de comparaison $(1 \mathrm{ppm}=0,0001 \%$ ou $0,1 \%=1000$ ppm) (tableau : B. Gratuze).

Figure 11: Average compositions of the Campu Stefanu glass beads expressed in oxide weight percent for the main oxides ( $\mathrm{Na}, \mathrm{O}$ to $\mathrm{CuO}$ ) and in part per million for the other elements. Measured values for Corning $A(C O R N A)$ reference glass are also given $(1 p p m=0.0001 \%$ or $0.1 \%=$ $1000 p p m)$. peut être fixée autour du XIII ${ }^{\mathrm{e}}$ siècle avant J.-C. ${ }^{5}$, soit vers la fin du Bronze moyen selon la périodisation insulaire (PecheQuilichini, 2013) ${ }^{6}$, en accord avec le mobilier céramique associé au sein de l'US 118 et ailleurs sur le site (Cesari et al., 2011 ; 2012a). On rappellera ici que la question de l'arrivée en Corse de mobiliers exotiques avait déjà été soulevée à l'occasion de la découverte de plusieurs perles isolées en verre $^{7}$, puis à la suite de la reconnaissance du lingot de cuivre " peau de bœuf " de Sant'Anastasia (Borgo, Haute-Corse) (Lo Schiavo, 2006, 2013a, 2013b). L'individualisation de ces pièces avait conduit à penser à des formes de contact direct ou indirect (via la Sardaigne?) entre les insulaires et

5. La datation radiométrique Poz-55584, $3010 \pm 30 \mathrm{BP}$ soit 1325-1219 Cal. BC, soit une date calendaire de $1272 \pm 53 \mathrm{BP}$, avec une probabilité de $68 \%$, a été obtenue sur charbon de bois associé aux parures.

6. Le XIII ${ }^{\mathrm{e}}$ siècle correspond terminologiquement à la phase baptisée "Bronze récent " par superposition chronologique avec les modèles italiens de périodisation.

7. À Filitosa (Sollacaro, Corse-du-Sud), Foce (Moca-Croce, Corse-duSud) et Tiresa (Sartène, Corse-du-Sud) (Gratuze et al., 2007). des navires venus de Méditerranée orientale. Ces remarques venaient ainsi confirmer des faits observés depuis près d'un demi-siècle en Sardaigne (Ferrarese-Ceruti, 1979; 1980; Lo Schiavo et Vagnetti, 1993; Usai, 2007; Cucuzza, 2009; Serchisu, 2009; Pompiani et Sorro, 2011; Santoni et al., 2012; Usai et al., 2012) et dans toute la Méditerranée centrale (entre autres références : Vagnetti, 1999; Tanasi, 2001, 2010 ; Cazzella et Recchia, 2009; Cultraro, 2010; Tanasi et Vella, 2014), documentant la circulation de marchandises et l'assimilation de savoir-faire égéens (cycladiques, minoens puis surtout mycéniens), égyptiens, chypriotes, phéniciens, puis eubéens, entre le $\mathrm{XVI}^{\mathrm{e}}$ et le $\mathrm{VIII}^{\mathrm{e}}$ siècle avant notre ère. La découverte des parures de Campu Stefanu apporte des informations nouvelles sur les circuits d'approvisionnement en denrées semi-précieuses. L'association stricte de l'ambre baltique, probablement mise en forme en Grèce continentale, à du verre proche-oriental ayant servi à réaliser des perles de type égyptien montre la complexité des circonstances ayant permis la convergence des artefacts dans le sud-ouest 


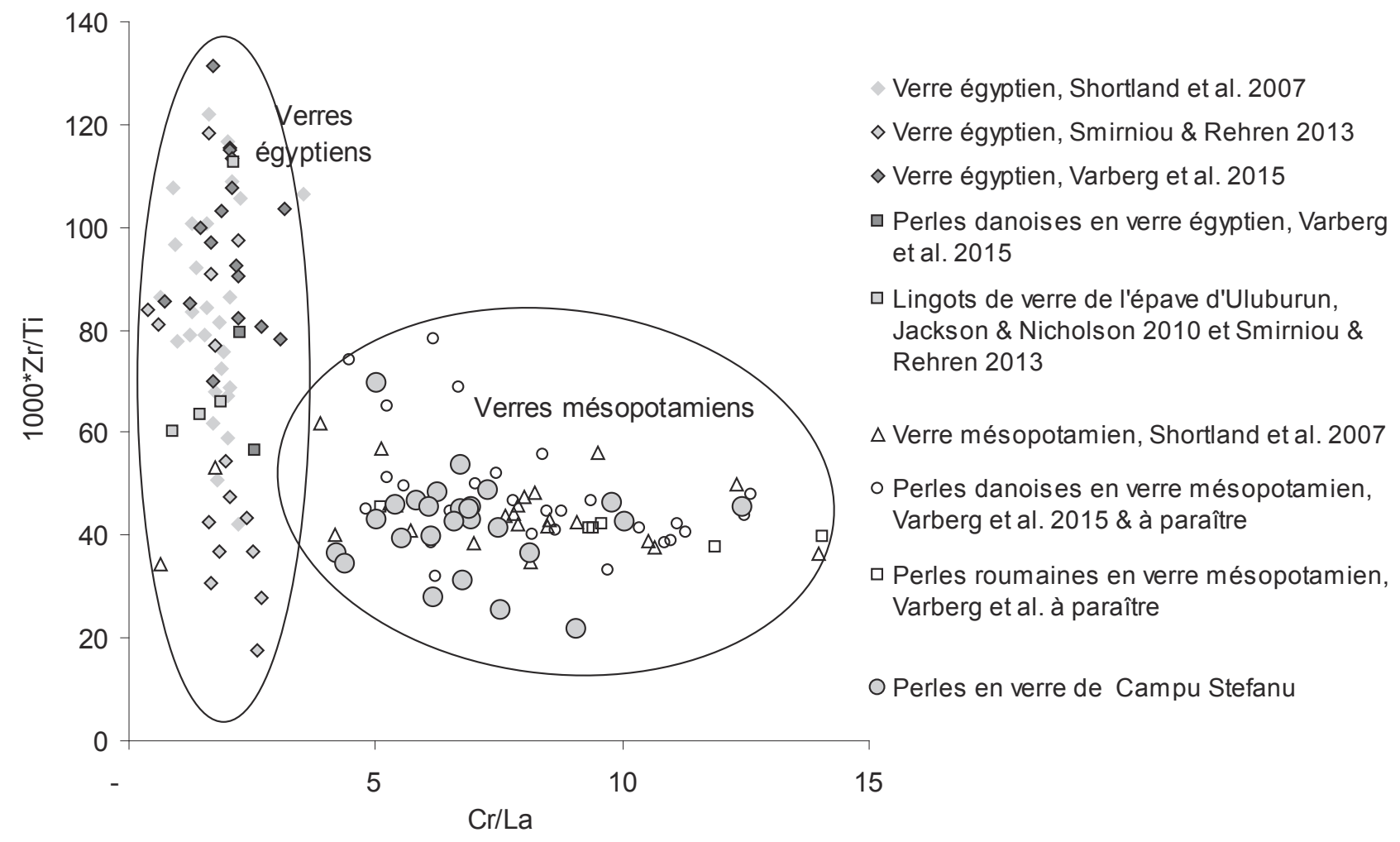

Figure 12 : Comparaison des rapports de concentrations chrome/lanthane et zirconium/titane des perles en verre de Campu Stefanu avec des corpus égyptiens et mésopotamiens de référence (DAO : B. Gratuze).

Figure 12: Comparison of the chromium/lanthanum and zirconium/titanium concentration ratios of the Campu Stefanu glass beads with reference groups of Egyptian and Mesopotamian glasses.

de la Corse, à travers un nombre d'intermédiaires indéterminé. En l'état des connaissances, l'hypothèse de trajectoire la plus crédible peut être conçue à partir de l'interprétation morphologique des perles en ambre, qui traduirait une conception du collier sur le territoire mycénien ${ }^{8}$, peut-être déjà avec les éléments vitreux originaires de Mésopotamie. L'ensemble a donc vraisemblablement été assemblé en Grèce sud-occidentale, la région qui fournit en moyenne le plus de preuves de contacts avec la Méditerranée occidentale, par l'intermédiaire de l'installation de nombreux artisans dans les Pouilles et en Calabre?. L'attribution chronologique du dépôt de l'abri 1, vers le xiII ${ }^{\mathrm{e}}$ siècle avant notre ère, correspond à l'Helladique récent IIIB (LHIIIB). À la vue de la multiplicité potentielle des routes empruntées par les vec-

8. Un travail de l'ambre brut dans d'autres régions, telles la Sardaigne ou l'Italie méridionale, ne peut toutefois être totalement exclu (Blake, sous presse).

9. Voir par exemple : Buxeda et al., 2003 ou Jones et al., 2005 pour les phénomènes de transfert artisanal dans le domaine céramique. L'hypothèse d'une origine égéenne, dans le cadre d'associations ambre-verre, au Bronze moyen, est aujourd'hui collégialement acceptée en Méditerranée occidentale (Bellintani, 2010; Bellintani et al., 2012 et bibliographie relative). teurs mycéniens en Méditerranée occidentale (figure 13), la possibilité d'une introduction indirecte et décalée en Corse n'est pas à exclure.

\section{Conclusion et Perspectives}

Les perles de Campu Stefanu retrouvées en connexion (probablement issues d'un collier) constituent une découverte exceptionnelle. D'une part en raison de leur contexte chronologique bien documenté, d'autre part par le nombre de perles en verre et la présence exceptionnelle de perles résineuses, ce dernier matériau étant rarement conservé dans ces contextes. Les origines des matériaux, au Levant pour le verre, sur les rivages baltiques pour l'ambre, soulignent des circulations aux échelles européenne et méditerranéenne avec, d'après la typologie, un transit par le monde égéen avant d'arriver en Corse.

La question de la présence d'objets exotiques dans cette sépulture corse du milieu de l'âge du Bronze renvoie vraisemblablement à la circulation et aux pratiques d'échange des 


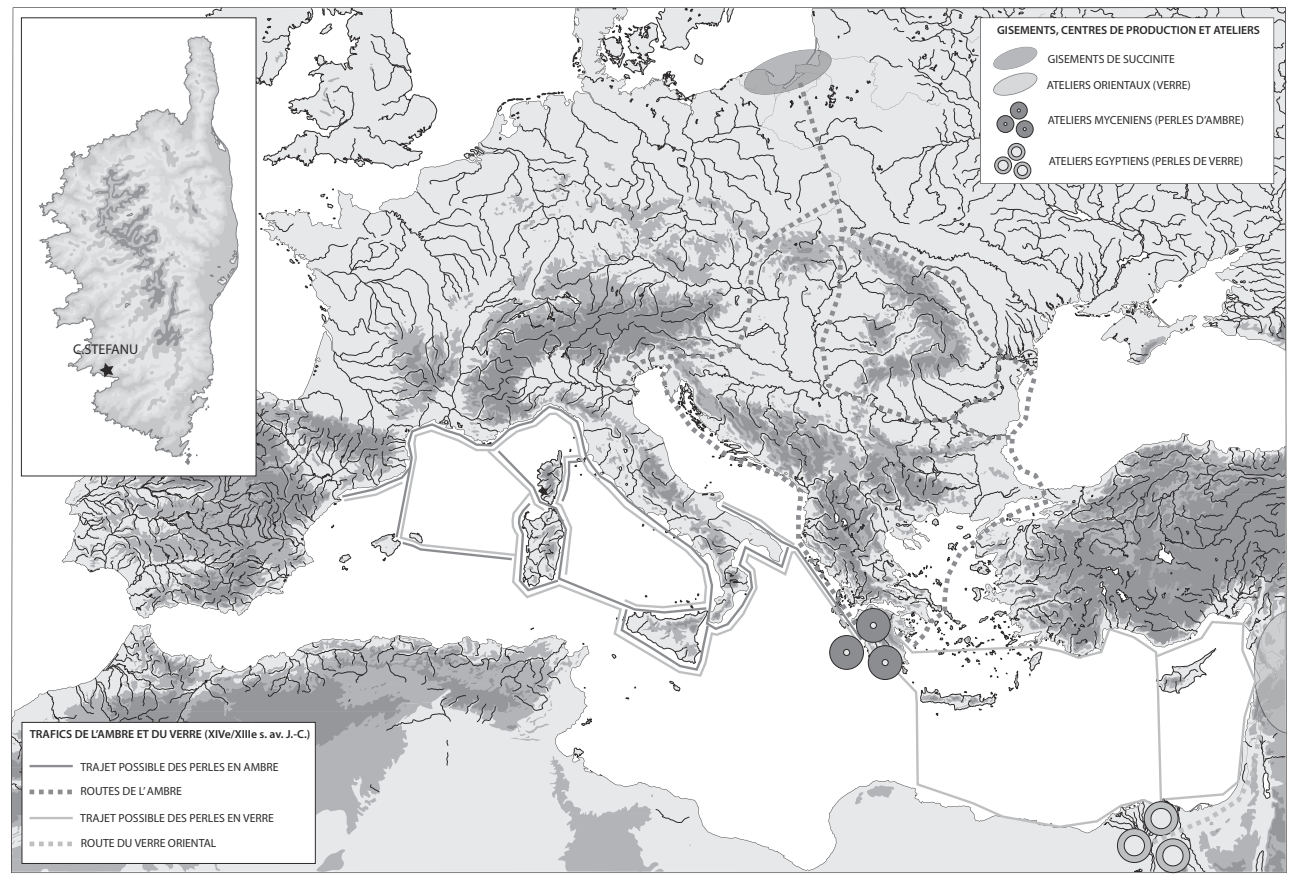

Figure 13: (Voir planche couleur IX) Hypothèse de reconstitution des gisements de matières premières, des ateliers et des axes de circulation entrant en considération dans le cadre des éléments constituant le collier (DAO : K. Peche-Quilichini).

Figure 13: (See colour plate IX) Hypothetical reconstruction of raw materials deposits, workshops and circulation routes entering into consideration as part of the elements constituting the necklace. biens de prestige et des exotica (Brysbaert et Vetters, 2013) entre potentats locaux. De telles pratiques sont attestées dans toute la Méditerranée orientale à partir du II ${ }^{\mathrm{e}}$ millénaire av. J.-C. (Lerouxel, 2002; Gestoso Singer, 2006; 2008) et envisagées en Occident, notamment en Sardaigne, à partir d'une phase avancée du Bronze moyen (Lo Schiavo et Vagnetti, 1993; Bellintani et al., 2006; Radina et Recchia, 2006). Dans ce cadre, il convient de souligner que les découvertes de parures en ambre et/ou en verre dans des contextes du Bronze moyen nuragique proviennent, comme c'est le cas à Campu Stefanu, essentiellement de sépultures ${ }^{10}$ (Atzeni $e t$ al., 2012; Bellintani et Usai, 2012; Bellintani et al., 2012). Si ce phénomène ne peut être interprété plus en profondeur à l'heure actuelle en raison de l'extrême complexité des cas de figure envisageables (Jung et Mehofer, 2013), il permet de mieux comprendre la place de la Corse dans les réseaux d'approvisionnement (et sur l'échiquier politico-culturel?) issus d'Orient et impactant le bassin méditerranéen occidental. L'insertion de ces éléments exogènes au sein d'un monde où ils paraissent rares voire rarissimes et ce, malgré un nombre accru de découvertes ces dernières années, autorise en outre à fournir une image plus précise de leur statut vis-à-vis (et au sein) des systèmes matériels corses et sardes (figure 14). Si elle ne se pose pas directement ici, la question de l'influence technico-fonctionnelle de ces importations

10. À l'exception du nuraghe Antigori, pour le Bronze moyen/récent (Ferrarese-Ceruti, 1980). sur les industries locales constitue une problématique qui a d'ailleurs récemment connu des développements tout à fait originaux (Graziani et al., 2014) et susceptibles de lui apporter des résonnances sociales. La découverte, en 2015, d'un fragment de lingot cuivre-plomb en contexte stratifié sur le site de Cuciurpula ${ }^{11}$ vient confirmer l'intérêt d'une démarche scientifique qui offre des perspectives nombreuses, qui feront prochainement l'objet d'une publication synthétique (Peche-Quilichini et al., 2016).

\section{Remerciements}

Nous tenons à remercier Marilou Nordez (université Toulouse 2 Jean-Jaurès), Peter Van Dommelen (Brown University), Mateusz Jaeger (Poznań Adam Mickiewicz University) et les deux rapporteurs anonymes pour les efficaces relectures externes, ainsi qu'Emma Blake (University of Arizona) et Samuel Guérin (INRAP) pour la mise à disposition de publications et documentations inédites.

11. Dans l'habitation 26 (direction des travaux : T. Lachenal). 


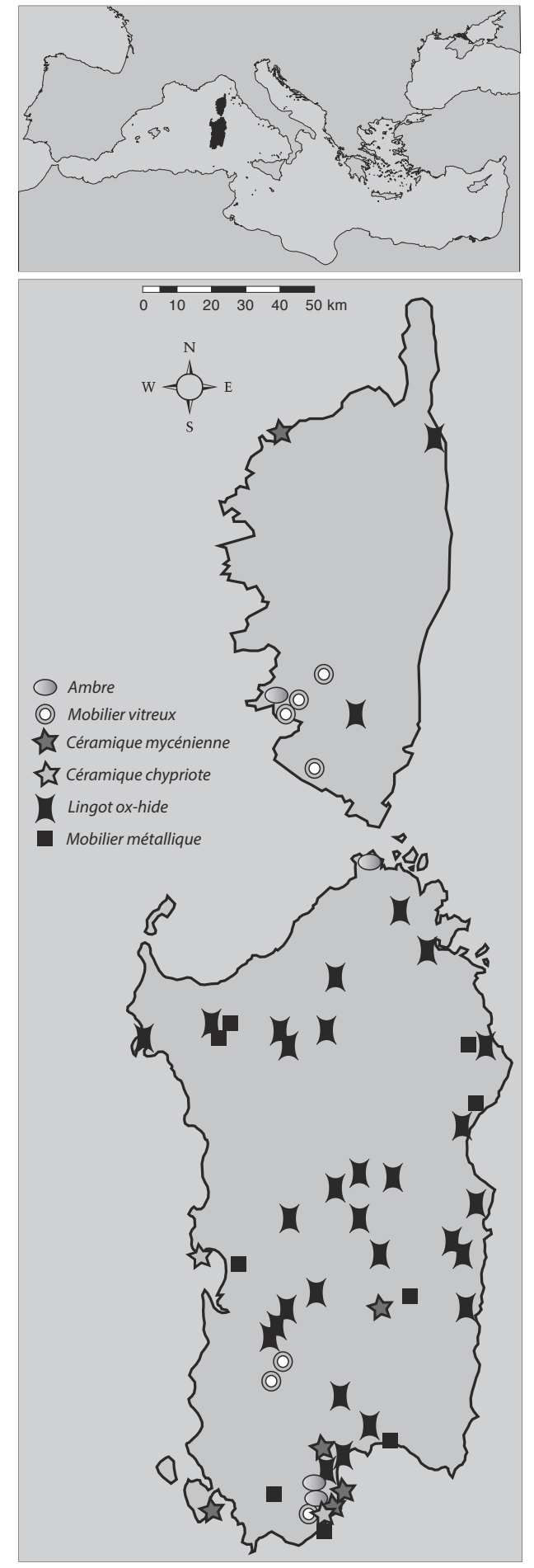

Figure 14 : Distribution géographique des mobiliers d'importation orientale en Corse et en Sardaigne durant la seconde moitié du Bronze moyen (DAO : K. Peche-Quilichini).

Figure 14: Geographical distribution in Corica and Sardinia of oriental importation artifacts for the second part of the $M B A$.

\section{Bibliographie}

Angelini I., Bellintani P., 2005. Archaeological ambers from Northern Italy: an FTIR-DRIFT study of provenance by comparison with the geological amber database. Archaeometry, 47, p. 441-454.

Atzeni E., Usai A., Bellintani P., Fonzo O., Lai L., Tyкot R., Setzer T.J., Congiu R., Simbula S., 2012. Le tombe megalitiche nuragiche di Sa Sedda 'e sa Caudela (Collinas - CA). In Preistoria e Protostoria della Sardegna. Atti della XLIV Riunione Scienfica dell'Istituto Italiana di Preistoria e Protostoria (Cagliari-Barumini-Sassari, novembre 2009). Florence, IIPP, p. 665-670.

Beck C.W., Beck L.Y., 1995. Analysis and Provenience of Minoan and Mycenaean Amber, V: Pylos and Messenia. Greek, Roman and Byzantine Studies, 36, p. 119-135.

Bellintani P., 2010. Ambra. Una materia prima dal nord (ma non solo). In A. Cazzella, G. Recchia (éd.). Ambra per Agamemnone. Indigeni e Micenei tra Egeo, Ionio e Adriatico nel II millenio a.C. Catalogo della mostra. Bari, Museo Civico Storico, p. 139-144.

Bellintani P., Usai A., 2012. Materiali vetrosi protostorici della Sardegna: inquadramento crono-tipologico e considerazioni sulle relazioni tra Mediterraneo centrale e orientale. In Preistoria e Protostoria della Sardegna. Atti della XLIV Riunione Scienfica dell'Istituto Italiana di Preistoria e Protostoria (Cagliari-Barumini-Sassari, novembre 2009). Florence, IIPP, p. 1122-1130.

Bellintani P., Angelini I., Artioli G., Polla A., 2006. Origini dei materiali vetrosi italiani: esotismi e localismi. In Preistoria e Protostoria della Sardegna. Atti della XLIV Riunione Scienfica dell'Istituto Italiana di Preistoria e Protostoria (CagliariBarumini-Sassari, novembre 2009). Florence, IIPP, p. 14951531.

Bellintani P., Usai A., Fadda M.A., 2012. Ambre protostoriche della Sardegna: nuovi dati su tipologia e possibili indicatori di lavorazione locale. In Preistoria e Protostoria della Sardegna. Atti della XLIV Riunione Scienfica dell'Istituto Italiana di Preistoria e Protostoria (Cagliari-Barumini-Sassari, novembre 2009). Florence, IIPP, p. 1163-1171.

Bennet J., 2008. Palace TM: Speculations on palatial production in Mycenaean Greece with (some) reference to glass. In C.M. Jackson, E. Wager (éd.). Vitreous Materials in the Late Bronze Age Aegean. Sheffeld Studies in Aegean Archaeology. Oxford, Oxbow Books, p. 151-172.

Billaud Y., Gratuze B., 2002. Les perles en verre et en faïence de la Protohistoire française, In J. Guilaine (éd). Matériaux, productions, circulation, du Néolithique à l'Age du Bronze. Séminaires du Collège de France, Paris, Errance, p. 193-212.

Blake E., sous presse. Late Bronze Age Sardinia: Acephalous Cohesion. In B. Knapp, P. van Dommelen (éd.). The 
Cambridge Prehistory of the Bronze and Iron Age Mediterranean. New York, Cambridge University Press.

Brysbaert A., Vetters M., 2013. A moving story about exotica: objects' long-distance production chains and associated identities at Tiryns, Greece. Opuscula. Annual of the Swedish Institutes at Athens and Rome, 6, p. 175-210.

Buxeda i Garrigós J., Jones R.E., Kilikoglou V., Levi S.T., Maniatis Y., Mitchell J., Vagnetti L., Wardle K.A., Andreou S., 2003. Technology transfer at the periphery of the Mycenaean world: the cases of Mycenaean pottery found in central Macedonia (Greece) and the plain of Sybaris (Italy). Archaeometry, 45, p. 263-284.

Cazzella A., Recchia G., 2009. The 'Mycenaeans' in the central Mediterranean: a comparison between the Adriatic and the Tyrrhenian seaways. Pasiphae, III, p. 27-40.

Cesari J., 1989a. Torre et castelli a torra. Contribution à l'étude des habitats de l'Age du Bronze de la Corse du Sud. Bulletin de la Société des Sciences Historiques et Naturelles de la Corse, 656, p. 345-372.

Cesari J., 1989b. Contribution à l'étude des habitats de l'Age du Bronze de la Corse-du-Sud. In A. D’Anna, X. Gutherz (éd.). Enceintes, habitats ceinturés, sites perchés du Néolithique au Bronze ancien dans le sud de la France et les régions voisines (Lattes et Aix-en-Provence, avril 1987). Mémoires de la Société Languedocienne de Préhistoire, 2, p. 69-83.

Cesari J., 1992. Contribution à l'étude des habitats de l'Age du Bronze de la Corse du Sud. In La Sardegna nel Mediterraneo tra il Bronzo medio e il Bronzo recente (XVI-XII sec. a. C.). Atti del III $^{\circ}$ Convegno di Studi (Selargius-Cagliari, novembre 1987). Cagliari, Della Torre, p. 379-398.

Cesari J., Bressy C., Demouche F., Leandri F., Nebbia P., Peche-Quilichini K., 2011. Découverte récente d'un habitat pré- et protohistorique dans la basse vallée du Taravo : Campu Stefanu (Sollacaro, Corse-du-Sud). In I. Sénépart, T. Perrin, E. Thirault, S. Bonnardin (éd.). Marges, frontières et transgressions, Actualités de la recherche. Actes des VIII ${ }^{\text {es }}$ Rencontres Méridionales de Préhistoire Récente (Marseille, novembre 2008). Toulouse, AEP, p. 265-281.

Cesari J., Leandri F., Nebbia P., Peche-Quilichini K., Bressy C., Demouche F., 2012a. Note préliminaire sur l'habitat pré- et protohistorique de Campu Stefanu (Sollacaro, Corsedu-Sud). In Preistoria e Protostoria della Sardegna. Atti della XLIV Riunione Scientifica dell'Istituto Italiano di Preistoria e Protostoria (Cagliari-Barumini-Sassari, novembre 2009). Florence, IIPP, p. 435-454.

Cesari J., Leandri F., Nebbia P., Peche-Quilichini K., 2012 b. Note préliminaire sur le Néolithique cardial de l'abri « $\mathrm{N}^{\circ} 1$ " du gisement de Campu Stefanu (Sollacaro, Corse-du-Sud). In C. del Vais (éd.). Epi oinopa ponton. Studi sul Mediterraneo antico in ricordo di Giovanni Tore. Oristano, S'Alvure, p. 79-96.
Courtaud P., Cesari J., Leandri F., Nebbia P., Perrin T., Petersen H.C., Zémour A., 2014. La sépulture mésolithique de Campu Stefanu (Sollacaro, Corse-du-Sud, France). In I. Sénépart, F. Leandri, J. Cauliez, T. Perrin, E. Thirault (ed.). Chronologie de la Préhistoire Récente dans le Sud de la France. Acquis 1992-2012. Actualités de la recherche. Actes des Xes Rencontres Méridionales de Préhistoire Récente (Ajaccio, octobre 2012). Toulouse, AEP, p. 275-288.

Cucuzza N., 2009. La ceramica micenea. In J. Bonetto, G. Falezza, A.R. Ghiotto (éd.). Nora. Il foro romano. Storia di un'area urbana dall'età fenicia alla tarda Antichità (19972006), II-1 : I materiali preromani. Padoue, Università di Padova, p. 3-5.

Cultraro M., 2007. Evidence of Amber in Bronze Age Sicily: Local Sources and the Balkan-Mycenaean Connection. In I. Galanaki, H. Tomas, Y. Galanakis, R. Laffineur (ed.), Between the Aegean and Baltic Seas: Prehistory Across Borders. Proceedings of the International Conference Bronze and Early Iron Age Interconnections and Contemporary Developments between the Aegean and the Regions of the Balkan Peninsula, Central and Northern Europe (Zagreb, Avril 2005). Aegaeum, 27, p. 377-389.

Cultraro M., 2010. Imitazioni di forme egeo-micenee nel Bronzo medio a Catania? In M.G. Branciforti, V. La Rosa (éd.). Tra lava e mare. Contributi all'archaiologhia di Catania. Catane, Le Nove Muse, p. 73-80.

Daher C., Paris C., Le Hô A.-S., Bellot-Gurlet L., Echard J.-P., 2010. A joint use of Raman and Infrared spectroscopies for the identification of natural organic media used in ancient varnishes. Journal of Raman Spectroscopy, 41, p. 1204-1209.

Daher C., Bellot-Gurlet L., Le Hô A.-S., Paris C., Regert M., 2013. Advanced discriminating criteria for natural organic substances of Cultural Heritage interest: Spectral decomposition and multivariate analyses of FT-Raman and FT-IR signatures. Talanta, 115, p. 540-547.

Du Gardin C., 1986. La parure d'ambre à l'Âge du Bronze en France. Bulletin de la Société Préhistorique Française, 83, p. 546558.

Ferrarese-Ceruti M.L., 1979. Ceramica micenea in Sardegna (notizia preliminare). Rivista di Scienze Preistoriche, XXXIV, p. 243-253.

Ferrarese-Ceruti M.L., 1980. Micenei in Sardegna! In Micenei in Sardegna?, Rome, Atti della Riunione dell'Accademia Nazionale dei Lincei, p. 391-393.

Gaslain C., Casanova M., 2009. L'ambre en Méditerranée à l'âge du Bronze. In J.-R. Gaborit (éd.). Circulation des matières premières en Méditerranée, transferts de savoirs et de techniques. Paris, CTHS, p. 89-100. 
Gestoso Singer G., 2006. El intercambio de materias primas y bienes de prestigio entre Egipto y los estados de Mesopotamia (siglos XV y XIV a.C.). Aula Orientalis, 2, p. 189-211.

Gestoso Singer G., 2008. Amber in the ancient Near East. I-Medjat, 2, p. 2-4.

Gratuze B., 2013. Glass Characterisation Using Laser Ablation Inductively Coupled Plasma Mass Spectrometry Methods. In K. Janssens (éd.). Modern Methods for Analysing Archaeological and Historical Glass. Hoboken, John Wiley \& Sons Ltd, p. 201-234.

Gratuze B., 2014. Application de la spectrométrie de masse à plasma avec prélèvement par ablation laser (LA-ICP-MS) à l'étude des recettes de fabrication et de la circulation des verres anciens. In P. Dillmann, L. Bellot-Gurlet (éd.), Circulation des matériaux et des objets dans les sociétés anciennes, Collection Sciences Archéologiques, Paris, Archives Contemporaines, p. 259-291.

Gratuze B., Dussubieux L., Cesari J., Nebbia P., Magdeleine J., Pasquet A., Ottaviani J.-C., Billaud Y., 2007. La circulation des objets de parure en verre dans le Bassin Méditerranéen au cours de la Protohistoire : origine des perles retrouvées sur des sites corses de l'Age du Bronze et de l'Age du Fer. In A. D’Anna, J. Cesari, L. Ogel, J. Vaquer (éd.). Corse et Sardaigne préhistoriques. Actes du colloque du CTHS (Bastia, avril 2003). Paris, CTHS, p. 359-369.

Gratuze B., Koenig M.-P., Plouin S., Treffort J.-M., 2013. Les perles en faïence et en verre de l'âge du Bronze : contextes archéologiques et analyses pour l'Alsace et la Lorraine. Cahiers Alsaciens d'Archéologie, d'Art et d'Histoire, 56, p. 11-36.

Graziani J., Peche-Quilichini K., Paolini-Saez H., Soula F., 2014. Contribution à l'étude des paléométallurgies corses : les matrices pour le repoussé. In I. Sénépart, F. Leandri, J. Cauliez, T. Perrin, E. Thirault (ed.). Chronologie de la Préhistoire Récente dans le Sud de la France. Acquis 1992-2012. Actualités de la recherche, Actes des $\mathrm{X}^{\text {es }}$ Rencontres Méridionales de Préhistoire Récente (Ajaccio, octobre 2012). Toulouse, AEP, p. 447-457.

GrosjEan R., 1958. Balestra et Foce, monuments circulaires mégalithiques de la moyenne vallée du Taravo. Gallia Préhistoire, 1, p. 1-38.

Hughes-Brock H., 1998. Mycenaean Amber beads and ornaments: what can we learn from their shapes and from one another? In Peretto, C., Giunchi, C., Fabro, M.(ed.), Atti del XIII congresso dell'UISPP (Forli, 1996). ABACO, p. 491-496.

Hughes-Brock H., 2005. Amber and some other travellers in the Bronze Age Aegean and Europe. In A. Dakouri-Hild, S. Sherratt (ed.), Autochthon: Papers presented to O. T. P. $\mathrm{K}$. Dickinson on the occasion of his retirement (Londres, novembre 2005), BAR International Series, 1432. Oxford, Archaeopress, p. 301-316.
Jackson C., Nicholson P., 2010. The provenance of some glass ingots from the Uluburun shipwreck. Journal of Archaeological Science, 37, p. 295-301.

Jones R.E., Levi S.T., Bettelli M., 2005, Mycenaean pottery in the central Mediterranean: imports, imitations and derivatives. In R. Laffineur, E. Greco (éd.). Emporia. Aegeans in the central and eastern Mediterranean. Proceedings of the $10^{\text {th }}$ International Egean Conference (Athènes, avril 2014), II. Aegaeum, 25, p. 539-549.

Jung R., Mehofer M., 2013. Mycenaean Greece and Bronze Age Italy: cooperation, trade or war? Archäologisches Korrespondenzblatt, 43, p. 175-193.

Lerouxel F., 2002. Les échanges de présents entre souverains amorrites au XVIII ${ }^{\mathrm{e}}$ siècle av. n. è. d'après les archives royales de Mari. Florilegium Marianum, VI, p. 413-463.

Lo Schiavo F., 2006. Il Mediterraneo occidentale prima degli Etruschi. In Gli Etruschi e il Mediterraneo. Commerci e politica. Annali della Fondazione per il Museo "Claudio Faina", v. XIII. Orvieto, p. 29-58.

Lo Schiavo F., 2013a. Il lingotto di rame "a pelle di bue" da Sant'Anastasia (Borgo). In P. Pergola (éd.). Mariana et la Vallée du Golo. Actes du colloque international (Bastia-Lucciana, septembre 2004), Vol. II. Patrimoine d'une île / Patrimoniu isulanu, 3, p. 171-185.

Lo Schiavo F., 2013b. I lingotti "a forma di pelle di bue" da S. Anastasia (Borgo) e da Séte, nel quadro della problematica della navigazione e degli scambi nel Mediterraneo centrale. In P. Pergola, F. Lo Schiavo (éd.). Les Lingots " peau de bœuf » et la navigation en Méditerranée centrale. Actes du IIe Colloque International (Lucciana-Mariana, septembre 2005). Patrimoine d'une île / Patrimoniu isulanu, 4, p. 15-32.

Lo Schiavo F., Vagnetti L., 1993. Alabastron miceneo dal nuraghe Arrubiu di Orroli. Rendiconti dell'Accademia dei Lincei, 9, p. 121-148.

Maran J., 2013. Bright as the sun: the appropriation of amber objects in Mycenaean Greece. In H.P. Hahn, H. Weiss (éd.). Mobility, meaning and the transformations of things. Londres, Oxbow Books, p. 147-169.

Nakai I., Tantrakarn K., Kato N., Kawai N., Nishisaka A., Yoshimura S., 2009. XRF analysis of $16^{\text {th }}$ century BC transparent glass beads excavated from a hillside in northwest Saqqara, Egypt. In Annales of the 17th AIHV Congress (Anvers, 2006). Bruxelles, p. 27-31.

Néraudeau D., Manem S., Delclos X., Girard V., 2012. L'ambre crétacé des Charentes. Une alternative à l'ambre balte? In G. Marchand, G. Querré (éd.). Roches et sociétés de la Préhistoire. Entre massifs cristallins et bassins sédimentaires. Rennes, PUR, p. 265-271.

Onoratini G., Guiliano M., Mille G., Simon P., 2009. L'ambre albo-cénomanien de la montagne de Lure (Alpes-de-Haute-

ArcheoSciences, revue d'archéométrie, 40, 2016, p. 65-81 
Provence), outil stratigraphique et paléogéographique. Geobios, 42, p. 89-99.

Peche-Quilichini K., 2013. Chronologie, productions matérielles et dynamiques socio-culturelles : le point sur le séquençage de l'âge du Bronze de la Corse. In J. de Lanfranchi (éd.). Quoi de neuf en archéologie? Actes des XIIIe Rencontres Culturelles du Musée de l'Alta Rocca (Levie, novembre 2011). Levie, p. 33-77.

Peche-Quilichini K., 2014. Sous le tesson, l'alliage. Note morphologique et chronologique sur les creusets de l'âge du bronze de Corse. Bulletin de l'Association pour la Promotion de la Recherche sur l'Age du Bronze, 12, p. 129-133.

Peche-Quilichini K., Graziani J., Antolini J.-P., Gardella M.-A., Milletti M., 2014. Les matrices de fusion protohistoriques de Corse : état de la recherche et découvertes récentes. In I. Sénépart, F. Leandri, J. Cauliez, T. Perrin, E. Thirault (ed.). Chronologie de la Préhistoire Récente dans le Sud de la France. Acquis 1992-2012. Actualités de la recherche. Actes des Xes Rencontres Méridionales de Préhistoire Récente (Ajaccio, octobre 2012). Toulouse, AEP, p. 431-446.

Peche-Quilichini K., Bellot-Gurlet L., Cesari J., Gratuze B., Graziani J., Leandri F., Paolini-Saez H., 2016. From Shardania to Læstrygonia... Eastern origin prestige goods and technical transfers in Corsica through Middle and Final Bronze Age. In M. Fotiadis, R. Laffineur, Y. Lolos, A. Vlachopoulos (éd.). Hesperos. The Aegean seen from the West, Proceedings of the 16th International Aegean Conference (Ioannina, mai 2016). Aegaeum, Annales Liégeoises d'Archéologie Egéenne.

Perrichot V., Néraudeau D., Nel A., de Ploëg G., 2007. A reassessment of the Cretaceous amber deposits from France and their palaeontological significance. African Invertebrates, $48: 213-227$.

Plouin S., Koenig M.-P., Gratuze B., 2012. Les perles en verre de l'âge du Bronze d'Alsace et de Lorraine. In Le verre en Lorraine et dans les régions voisines. Actes du Colloque international, 26e Rencontres de l'AFAV (Metz, novembre 2011). Monographies Instrumentum, 42, p. 11-36.

Pompiani E., Sorro L., 2011. Nuove testimonianze micenee da Sulky (Sardegna). Rivista di Studi Fenici, XXXIX, p. 291-302.

Pulak C., 2008. The Uluburun Shipwreck and Late Bronze Age Trade. In Aruz, J., Benzel, K., Evans, J.M. (éd.). Beyond Babylon: Art, Trade, and Diplomacy in the Second Millennium B.C., The Metropolitan Museum of Art Exhibition Catalog, New York, p. 3999-4005.

Radina F., Recchia G., 2006. Scambi senza ceramica : ambra, avorio e pasta vitrea nei rapporti tra italia sud-orientale e mondo egeo. In Materie prime e scambi nella Preistoria italiana. Atti della XXXIX Riunione Scienfica dell'Istituto Italiana di Preistoria e Protostoria (Florence, novembre 2004). Florence, IIPP, p. 1555-1565.
Rehren Th., 2014. Glass Production and Consumption between Egypt, Mesopotamia and the Aegean. In P. Pfälzner, H. Niehr, E. Pernicka, S. Lange, T. Köster (éd.). Contextualizing Grave Inventories in the Ancient Near East. Qatna Studien Supplements, 3, Harrassowitz, Wiesbaden, p. 217-223.

Rehren T., Pusch E., 2005. Late Bronze Age Glass Production at Qantir-Piramesses, Egypt. Science, 308, p. 1756-1758.

Santoni V., Bacco G., Lo Schiavo F., 2012. Frammento di lingotto "a forma di pelle di bue" dal nuraghe Serucci, Gonnesa (Cagliari). In Preistoria e Protostoria della Sardegna. Atti della XLIV Riunione Scienfica dell'Istituto Italiana di Preistoria e Protostoria (Cagliari-Barumini-Sassari, novembre 2009). Florence, IIPP, p. 1583-1589.

Serchisu F., 2009. Considerazioni sulla circolazione del rame in Sardegna e nel Mediterraneo: i lingotti ox-hide. In M.G. Melis (éd.). Uomo e territorio. Dinamiche di frequentazione e di sfruttamento delle risorse naturali nell'Antiquità. Atti del convegno nazionale dei giovani archeologi (Sassari, septembre 2006). Muros, Nuova Stampa, p. 170-176.

Shortland A.J., 2005. The raw materials of early glasses: the implication of new LA-ICP-MS analyses. Annales $d u$ $16^{e}$ Congrès de l'Association Internationale pour l'Histoire du Verre, Nottingham, p. 1-5.

Shortland A.J., Eremin K., 2006. The analysis of second millennium glass from Egypt and Mesopotamia, Part 1: New WDS Analyses. Archaeometry, 48/4, p. 581-603.

Shortland A.J., Rogers N., Eremin K., 2007. Trace element discriminants between Egyptian and Mesopotamian Late Bronze Age glasses. Journal of Archaeological Science, 34, p. 781-789.

Smirniou M., Rehren T., 2013. Shades of blue-cobalt-copper coloured blue glass from New Kingdom Egypt and the Mycenaean world: a matter of production or colourant source? Journal of Archaeological Science, 40, p. 4731-4743.

TAnasi D., 2001. Considerazioni sulle influenze micenee nella cultura di Pantalica Nord: la produzione ceramica. Archivio Storico per la Sicilia Orientale, 97, p. 1-88.

TANAsi D., 2010. Bridging the gap. New data on the relationship between Sicily, the Maltese Archipelago and the Aegean in the Middle Bronze Age. Mare Internum, 2, p. 111-119.

Tanasi D., Vella C., 2014. Islands and mobility: exploring Bronze Age connectivity in the south-central Mediterranean. In P. Van Dommelen, B. Knapp (éd.). The Cambridge Prehistory of the Bronze and Iron Age Mediterranean. Cambridge, Cambridge University Press, p. 53-73.

Tite M., Shortland A.J., 2008. Production technology of faience and related early vitreous materials. Oxford University School of Archaeology Monograph, 72, Oxford. Oxbow Books Ltd.

Tite M., Shortland A.J., Hatton, G., Maniatis, Y., Kavoussanaki, D., Pyrli, M., Panagiotaki, M., 2008. The scientific examination of Aegean vitreous materials. Problems 
and potential. In C.M. Jackson, E. Wager (éd.). Vitreous Materials in the Late Bronze Age Aegean. Sheffield Studies in Aegean Archaeology. Oxford, Oxbow Books, p. 105-125.

Usai A., 2007. L'ambra nel percorso di sviluppo della Sardegna nuragica. In M.L. Nava, A. Salerno (éd.). Ambre. Transparenze dall'antico. Catalogo della mostra. Naples, Electa, p. 96-105.

Usai A., Loi C., Lo Schiavo F., 2012. Frammento di lingotto "a forma di pelle di bue" ed altri dal nuraghe Benezziddo di Aidomaggiore (Oristano). In Preistoria e Protostoria della Sardegna. Atti della XLIV Riunione Scienfica dell'Istituto Italiana di Preistoria e Protostoria (Cagliari-Barumini-Sassari, novembre 2009). Florence, IIPP, p. 1595-1600.

Vagnetti L., 1999. Mycenaean pottery in the central Mediterranean. Imports and local production in their context. In J.-P. Crielaard, V. Stissi, G.J. Van Wijingaarden (éd.). The complex past of pottery. Production, circulation and consumption of Mycenaean and Greek pottery, VIth to early Vth centuries B.C. (Amsterdam, Novembre 1996). Amsterdam, p. 137-155.

Varberg J., Gratuze B., Kaul F., 2015. Between Egypt, Mesopotamia and Scandinavia: Late Bronze Age glass beads found in Denmark. Journal of Archaeological Science, 64, p. 168-181.

Varberg J., Gratuze B., Kaul F., Haslund Hansen A., Rotea M., Wittenberg M., à paraître. Mesopotamian glass from Late Bronze Age Egypt, Romania, Germany, and Denmark. Journal of Archaeological Science.

Walton M.S., Shortland A., Kirk S., Degryse P., 2009. Evidence for the trade of Mesopotamian and Egyptian glass to Mycenaean Greece. Journal of Archaeological Science, 36, p. $1496-1503$. 


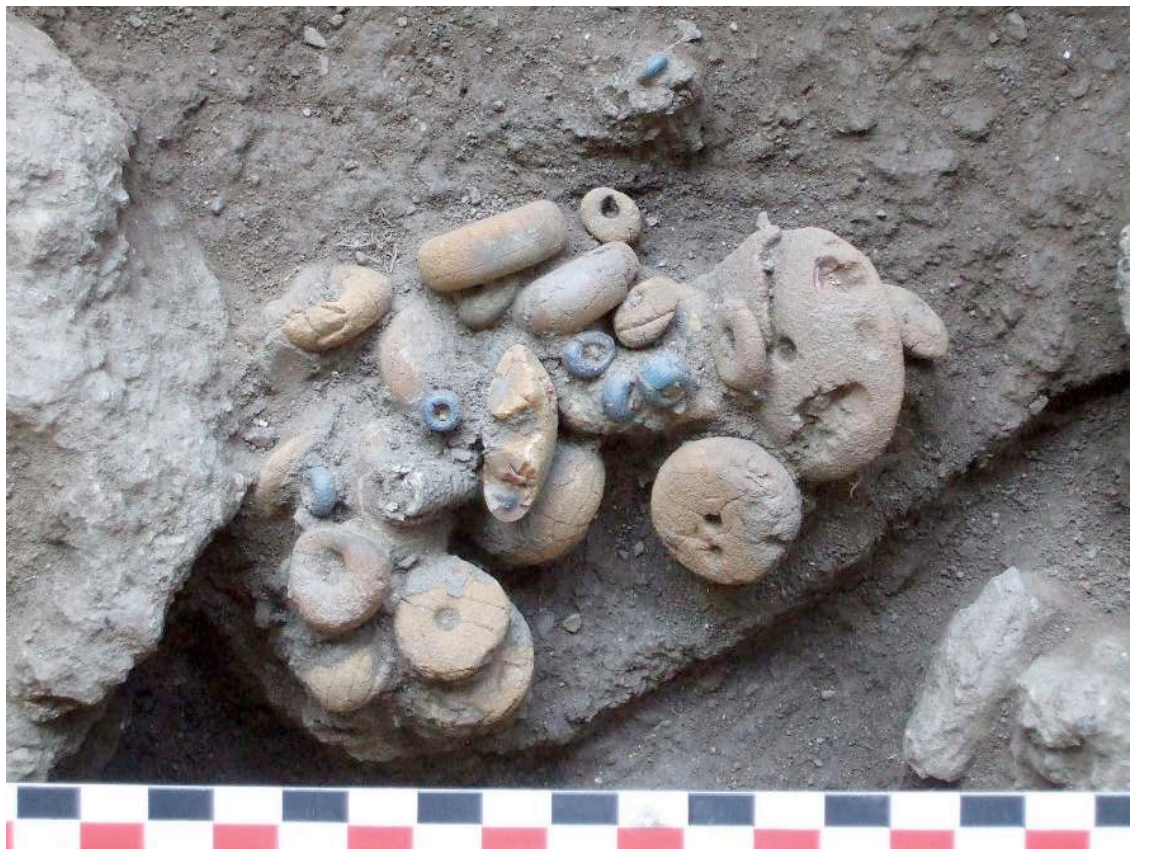

Figure 3 : Kewin Peche-Quilichini et al., Étude de provenance et implications économico-culturelles des parures vitreuses et résineuses du Bronze moyen de l'abri 1 de Campu Stefanu (Sollacaro, Corse-duSud) (p. 68)

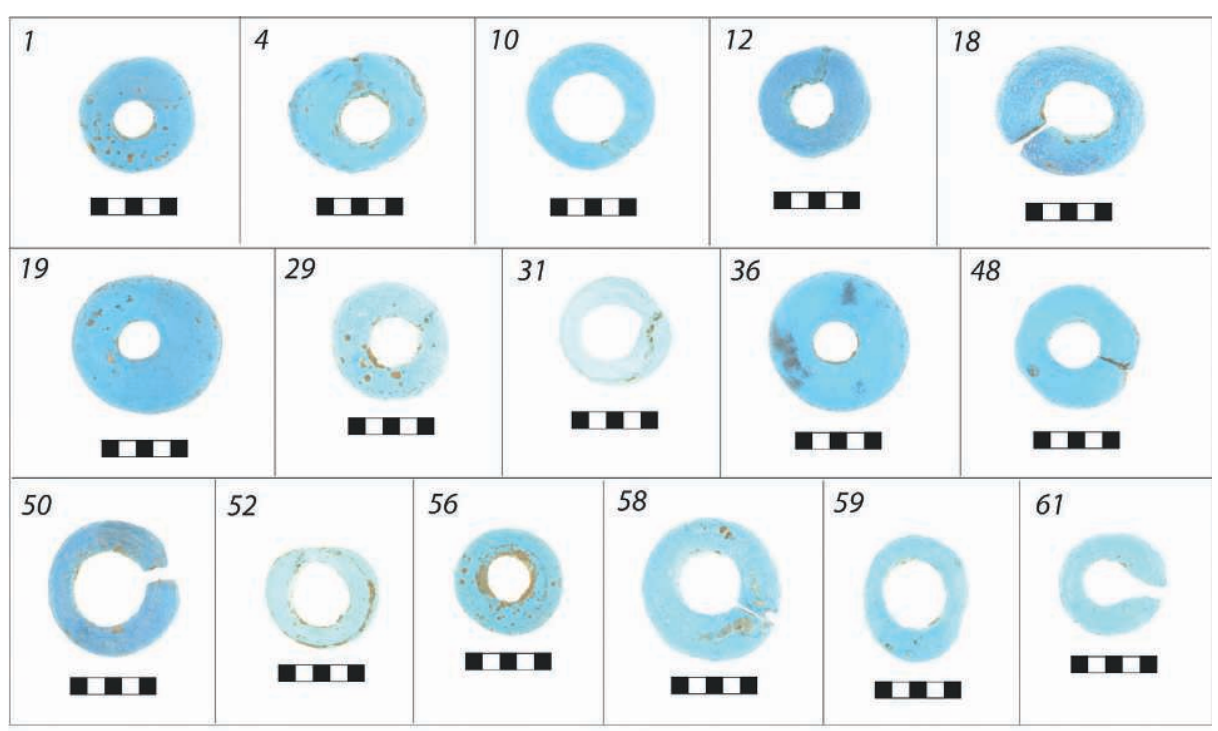

Figure 4 : Kewin Peche-Quilichini et al., Étude de provenance et implications économicoculturelles des parures vitreuses et résineuses du Bronze moyen de l'abri 1 de Campu Stefanu (Sollacaro, Corse-du-Sud) (p. 69)

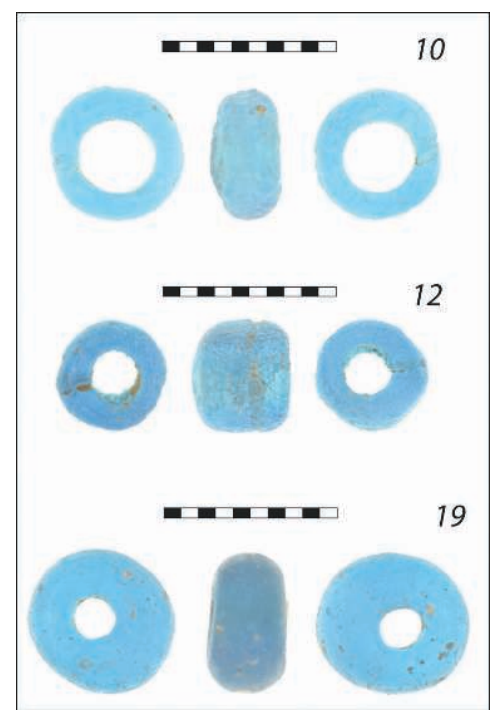

Figure 5 : Kewin Peche-QuiliCHINI et al., Étude de provenance et implications économico-culturelles des parures vitreuses et résineuses du Bronze moyen de l'abri 1 de Campu Stefanu (Sollacaro, Corsedu-Sud) (p. 69)
Figure 6 : Kewin Peche-Quilichin et al., Étude de provenance et implications économico-culturelles des parures vitreuses et résineuses du Bronze moyen de l'abri 1 de Campu Stefanu (Sollacaro, Corse-du-Sud) (p. 69)
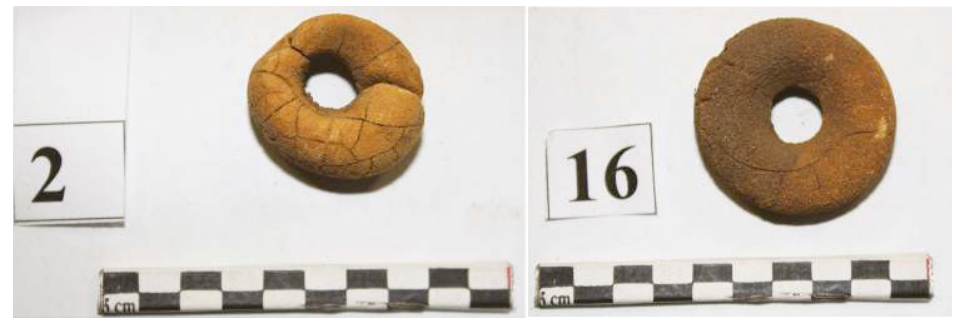


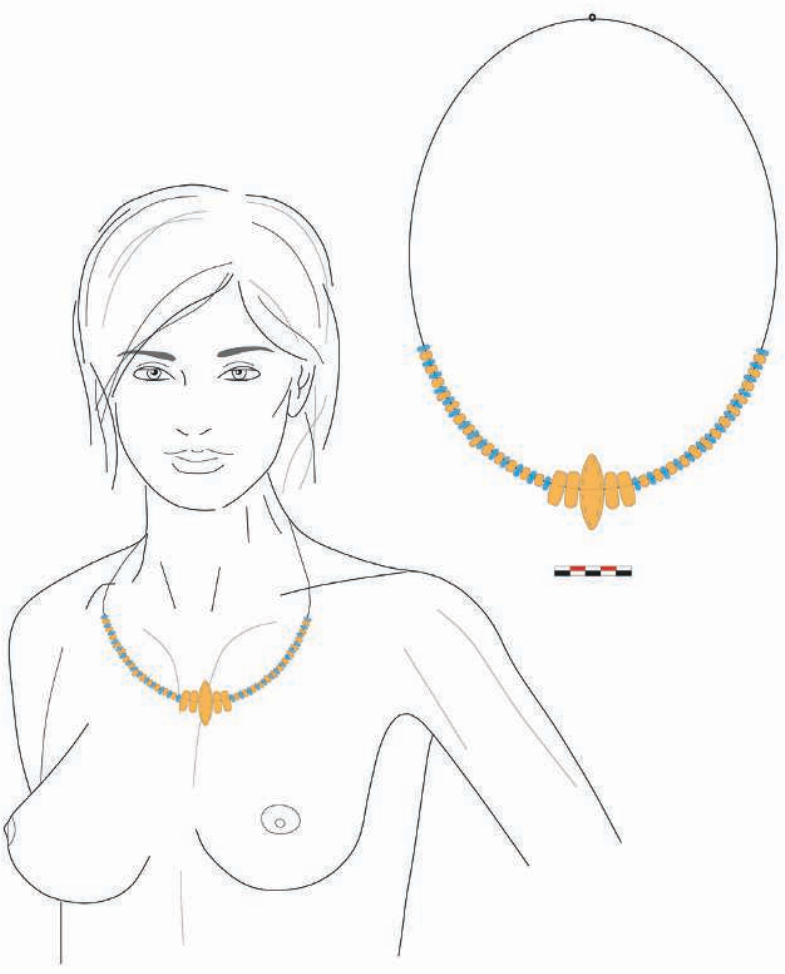

Figure 7 : Kewin Peche-Quilichini et al., Étude de provenance et implications économico-culturelles des parures vitreuses et résineuses du Bronze moyen de l'abri 1 de Campu Stefanu (Sollacaro, Corse-du-Sud) (p. 70)
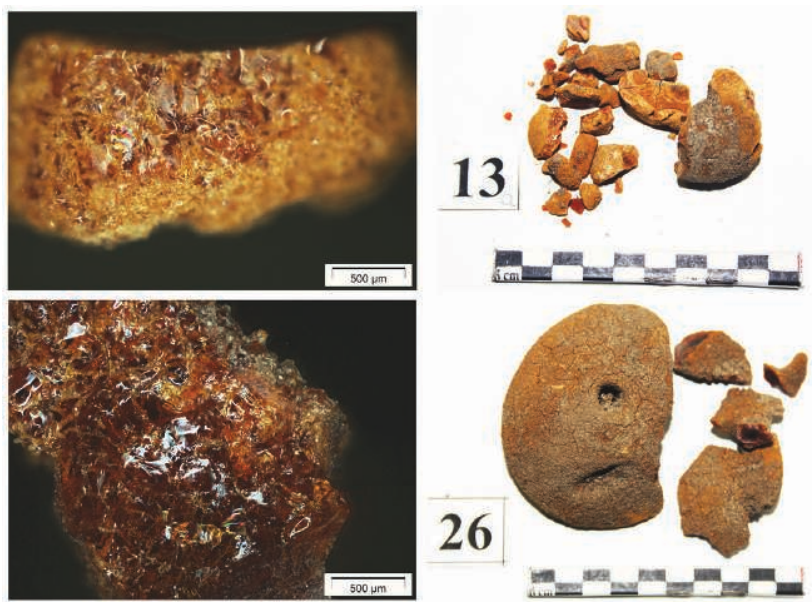

Figure 8 : Kewin Peche-Quilichini et al., Étude de provenance et implications économico-culturelles des parures vitreuses et résineuses du Bronze moyen de l'abri 1 de Campu Stefanu (Sollacaro, Corse-du-Sud) (p. 71)

Figure13 : Kewin Peche-Quilichini et al., Étude de provenance et implications économico-culturelles des parures vitreuses et résineuses du Bronze moyen de l'abri 1 de Campu Stefanu (Sollacaro, Corse-du-Sud) (p. 76)

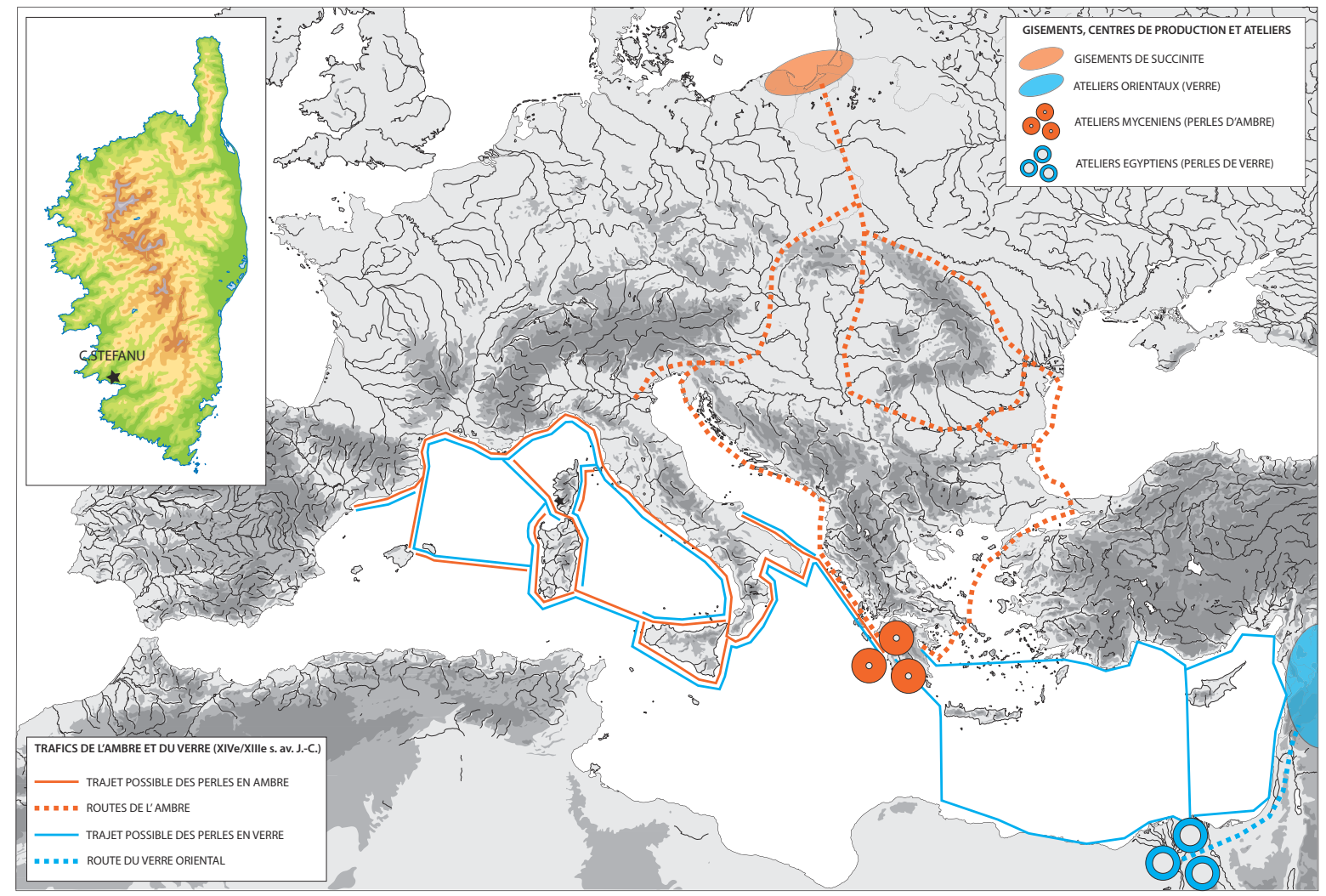

